\title{
UDP-sulfoquinovose formation by Sulfolobus acidocaldarius
}

\author{
Behnam Zolghadr • Bernhard Gasselhuber · Markus Windwarder • Martin Pabst • Daniel Kracher • \\ Martina Kerndl · Sonja Zayni - Andreas Hofinger-Horvath · Roland Ludwig • Dietmar Haltrich • \\ Chris Oostenbrink $\cdot$ Christian Obinger $\cdot$ Paul Kosma $\cdot$ Paul Messner $\cdot$ Christina Schäffer
}

Received: 4 September 2014 / Accepted: 5 January 2015 / Published online: 21 January 2015

(C) The Author(s) 2015. This article is published with open access at Springerlink.com

\begin{abstract}
The UDP-sulfoquinovose synthase Agl3 from Sulfolobus acidocaldarius converts UDP-D-glucose and sulfite to UDP-sulfoquinovose, the activated form of sulfoquinovose required for its incorporation into glycoconjugates. Based on the amino acid sequence, Agl3 belongs to the short-chain dehydrogenase/reductase enzyme superfamily, together with SQD1 from Arabidopsis thaliana, the only UDP-sulfoquinovose synthase with known crystal structure. By comparison of sequence and structure of $\mathrm{Agl} 3$ and SQD1, putative catalytic amino acids of Agl3 were selected for mutational analysis. The obtained data suggest for Agl3 a modified dehydratase reaction mechanism. We propose that in vitro biosynthesis of UDP-sulfoquinovose occurs through an $\mathrm{NAD}^{+}$-dependent oxidation/dehydration/ enolization/sulfite addition process. In the absence of a sulfur donor, UDP-D-glucose is converted via UDP-4-keto-Dglucose to UDP-D-glucose-5,6-ene, the structure of which
\end{abstract}

Communicated by S. Albers.

Electronic supplementary material The online version of this article (doi:10.1007/s00792-015-0730-9) contains supplementary material, which is available to authorized users.

\footnotetext{
B. Zolghadr $\cdot$ M. Kerndl $\cdot$ S. Zayni $\cdot$ P. Messner $(\square) \cdot$

C. Schäffer $(\bowtie)$

Department of NanoBiotechnology, NanoGlycobiology Unit,

Universität für Bodenkultur Wien, Muthgasse 11,

1190 Vienna, Austria

e-mail: paul.messner@boku.ac.at

C. Schäffer

e-mail: christina.schaeffer@boku.ac.at

B. Gasselhuber - M. Windwarder · M. Pabst .

A. Hofinger-Horvath $\cdot$ C. Obinger $\cdot$ P. Kosma

Department of Chemistry, Universität für Bodenkultur Wien,

Muthgasse 18, 1190 Vienna, Austria
}

was determined by ${ }^{1} \mathrm{H}$ and ${ }^{13} \mathrm{C}-\mathrm{NMR}$ spectroscopy. During the redox reaction the cofactor remains tightly bound to $\mathrm{Agl} 3$ and participates in the reaction in a concentrationdependent manner. For the first time, the rapid initial electron transfer between UDP-D-glucose and $\mathrm{NAD}^{+}$could be monitored in a UDP-sulfoquinovose synthase. Deuterium labeling confirmed that dehydration of UDP-D-glucose occurs only from the enol form of UDP-4-keto-glucose. The obtained functional data are compared with those from other UDP-sulfoquinovose synthases. A divergent evolution of Agl3 from S. acidocaldarius is suggested.

Keywords UDP-sulfoquinovose synthase Agl3 . Sulfolobus acidocaldarius · Enzyme mechanism . Site-directed mutagenesis $\cdot$ Sulfite

$\begin{array}{ll}\text { Abbreviations } & \\ \text { Agl3, SQD1 } & \text { UDP-sulfoquinovose synthases } \\ \mathrm{CD} & \text { Circular dichroism spectrometry } \\ \mathrm{COSY} & \text { Correlated spectroscopy } \\ \mathrm{D}_{2} \mathrm{O} & \text { Deuterium oxide } \\ \text { ESI MS } & \text { Electrospray ionization mass } \\ & \text { spectrometry }\end{array}$

Present Address:

M. Pabst

Department of Chemistry and Applied Biosciences,

ETH Zürich, HCI D 330 Vladimir-Prelog-Weg 1-5/10,

8093 Zurich, Switzerland

D. Kracher $\cdot$ R. Ludwig $\cdot$ D. Haltrich

Department of Food Science and Technology, Universität für

Bodenkultur Wien, Muthgasse 11, 1190 Vienna, Austria

C. Oostenbrink

Institute for Molecular Modeling and Simulation, Universität für

Bodenkultur Wien, Muthgasse 18, 1190 Vienna, Austria 


\begin{tabular}{|c|c|}
\hline GalE & UDP-glucose/galactose \\
\hline & 4-epimerase \\
\hline HMBC NMR & $\begin{array}{l}\text { Heteronuclear multiple bond } \\
\text { correlation nuclear magnetic } \\
\text { resonance spectroscopy }\end{array}$ \\
\hline HSQC & $\begin{array}{l}\text { Heteronuclear single quantum } \\
\text { coherence }\end{array}$ \\
\hline MilliQ & $\begin{array}{l}\text { 'Ultrapure' water (typically } \\
18.2 \mathrm{M} \Omega \cdot \mathrm{cm} \text { resistivity at } 25^{\circ} \mathrm{C} \text { ) }\end{array}$ \\
\hline $\mathrm{NAD}^{+} / \mathrm{NADH}$ & $\begin{array}{l}\text { Oxidized/reduced nicotinamide } \\
\text { adenine dinucleotide }\end{array}$ \\
\hline PGC & Porous graphitized carbon \\
\hline Quip6S & $\begin{array}{l}\text { Sulfoquinovose (6-deoxy-6-C- } \\
\text { sulfo-D-glucopyranose) }\end{array}$ \\
\hline RmlB & dTDP-D-glucose 4,6-dehydratase \\
\hline SDR & $\begin{array}{l}\text { Short-chain dehydrogenase/ } \\
\text { reductase enzyme superfamily }\end{array}$ \\
\hline SlaA & $\begin{array}{l}\text { Major surface (S-)layer protein } \\
\text { of Sulfolobus acidocaldarius }\end{array}$ \\
\hline TOCSY & $\begin{array}{l}\text { Total correlated NMR } \\
\text { spectroscopy }\end{array}$ \\
\hline UDP-D-glucose & Uridine $5^{\prime}$-diphospho-D-glucose \\
\hline UDP-D-glucose-5,6-ene & $\begin{array}{l}\text { UDP 6-deoxy-D-xylo-hex- } \\
\text { 5-enose }\end{array}$ \\
\hline
\end{tabular}

\section{Introduction}

UDP-sulfoquinovose is the nucleotide-activated form of sulfoquinovose (6-deoxy-6- $C$-sulfo-D-glucopyranose, Qui6S) and is required for the incorporation of sulfoquinovose into glycoconjugates. Among those is, for instance, the sulfolipid sulfoquinovosyl diacylglycerol which is found in the chloroplast membrane of plants and in cyanobacteria (Benning et al. 1993; Benning 1998; Riekhof et al. 2003; Sato et al. 2003; Shimojima 2011; Denger et al. 2014), with cyanobacterial strains of the genus Spirulina having recently gained interest because of the antiHIV properties of some of their sulfoquinovose-containing sulfolipids (Kwei et al. 2011). In the hyperthermophilic archaeon Sulfolobus acidocaldarius sulfoquinovose is either a component of the glycosylated, membrane-associated cytochrome $b$ complex (Zähringer et al. 2000), the major surface (S-) layer protein SlaA (Peyfoon et al. 2010), or the subunit of the archaellum filament FlaB (Meyer et al. 2013). Further reports on sulfoquinovose in archaea concern a so far uncharacterized oligosaccharide modifying the S-layer protein of Haloferax volcanii (Eichler 2013; Parente et al. 2014) and an operon encoding a sulfoquinovose synthase $(\mathrm{SqdB})$ in the haloarchaeon Haloquadratum walsbyi (Bolhuis et al. 2006).

Work on the sulfolipid biosynthesis in Arabidopsis thaliana identified SQD1 as the biosynthesis enzyme for
UDP-sulfoquinovose (Benning et al. 1993; Benning 1998), with SQD1 currently being the best-investigated UDP-sulfoquinovose-synthase. It shows high sequence similarity to sulfolipid biosynthesis enzymes of different organisms and to sugar nucleotide modifying enzymes such as UDP-glucose epimerase GalE (Thoden et al. 1996a; Liu et al. 1997) and dTDP-glucose-4,6-dehydratase (Gross et al. 2000; Allard et al. 2002). In a three-dimensional model of SQD1, which is based on the 1.8- $\AA$ crystallographic structure of UDP-glucose 4-epimerase (Liu et al. 1997) as a template, an $\mathrm{NAD}^{+}$binding site and active site interactions were predicted (Essigmann et al. 1999). The proposed reaction mechanism of SQD1 was confirmed after its crystallization at $1.6-\AA$ resolution in a complex with $\mathrm{NAD}^{+}$and the putative substrate UDP-D-glucose (Mulichak et al. 1999). The SQD1 protein has a bi-domain structure with a Rossmann fold for $\mathrm{NAD}^{+}$binding, revealing high structural similarity with the GalE enzyme (Thoden et al. 1996b). It is a member of the SDR family of enzymes (Kavanagh et al. 2008), with its structure showing conservation of the catalytic SDR amino acid residues. The Rossmann-fold fingerprint sequence at the pyrophosphate binding site of SDR enzymes is replaced by a G-XX-G-XX-G sequence in SQD1 (Mulichak et al. 1999), while the characteristic Y-XXX-K motif and a Ser/Thr residue are located at the active site of SQD1, to form the catalytic triad of SDR enzymes (Kavanagh et al. 2008) with Thr145, Tyr182, and Lys186 (Mulichak et al. 1999).

The proposed mechanism for SQD1 catalysis suggests that, in the absence of a sulfur donor, the reaction continues to the UDP-4-keto-glucose-5,6-ene product. At the active site of the enzyme, UDP-D-glucose and $\mathrm{NAD}^{+}$are bound, with the latter in the oxidized state. In a subsequent step, a sulfur donor would transfer sulfite to UDP-4-keto-glucose5,6-ene by a nucleophilic addition across the double bond, followed by reduction of the 4-keto group and regeneration of $\mathrm{NAD}^{+}$(Mulichak et al. 1999).

Characterization of SQD1 from A. thaliana (Sanda et al. 2001) showed that the highly purified enzyme exists as a complex with ferredoxin-dependent glutamate synthase (Shimojima et al. 2005), and the crystal structure of SQD1 showed that the $\mathrm{NAD}^{+}$cofactor is tightly bound to the $\mathrm{N}$-terminal domain of the enzyme (Mulichak et al. 1999). The main bottleneck for fully elucidating the mechanism of SQD1 was its low in vitro activity. Recombinant SQD1 expressed in Escherichia coli showed low in vitro activity as well (Sanda et al. 2001). Thus, the in vivo-mechanism of the sulfite transfer to C- 6 of UDP-D-glucose by UDPsulfoquinovose synthases is still unknown.

To elucidate the biosynthesis of UDP-sulfoquinovose in $S$. acidocaldarius, its genome was scanned for the presence of homologues of the bacterial $s q d B$ or eukaryal sqdl genes known to encode UDP-sulfoquinovose 
synthases (Meyer et al. 2011). The scan revealed Saci0423 with $\sim 40 \%$ sequence identity to SQD1 of A. thaliana. As shown for SQD1, Agl3 is a member of the SDR superfamily of enzymes (Field and Naismith 2003; Kavanagh et al. 2008). In a recent study, the agl3 (saci0423) gene was confirmed to code for the UDP-sulfoquinovose synthase involved in the biosynthesis of the $S$. acidocaldarius S-layer $\mathrm{N}$-glycan. Targeted deletion of agl3 impaired UDP-sulfoquinovose synthesis and resulted in a mutant lacking sulfoquinovose in its S-layer glycan (Meyer et al. 2011). In addition, the lack of agl3 resulted in a reduced molecular mass of FlaB, indicating that FlaB is also modified with the $\mathrm{N}$-glycan containing sulfoquinovose (Meyer et al. 2013). In close vicinity of $a g l 3$, several genes are localized which are predicted to code for carbohydrateactive enzymes linked to the UDP-sulfoquinovose metabolism. These include agl4 (saci0424), annotated as a glucokinase, predicted to provide glucose 1-phosphate as a substrate for the NDP-glucose pyrophosphorylase Agl2, encoded by agl2 (saci0422), which would generate UDPglucose, serving as the immediate substrate for Agl3. Eventually, agll (saci0421), coding for a membranebound glycosyltransferase, would be involved in the transfer of sulfoquinovose from UDP-sulfoquinovose to the $\mathrm{N}$-acetylglucosamine residue of the $\mathrm{N}$-glycan (Meyer et al. 2011).

In the present study, we investigate the in vitro activity of Agl3 and propose that different reaction products can be formed dependent on the type and the amount of substrate. A complex reaction cycle is followed, including oxidation, dehydration, enolization, and reprotonation of UDP-D-glucose. Point mutation studies have been performed by targeting amino acids known to be crucial for enzymatic activity of enzymes from the SDR superfamily (Field and Naismith 2003; Kavanagh et al. 2008). This study provides new insights into the reaction mechanism of Agl3 of S. acidocaldarius and additionally unveils possible evolutionary differences between the planta enzyme SQD1 and the prokaryotic UDP-sulfoquinovose synthase.

\section{Results and discussion}

Phylogenetic and functional analysis of Agl3

As a starting point the phylogenetic relationship of UDPsulfoquinovose synthases from archaea, (cyano)bacteria, and plants was analyzed. Agl3 from $S$. acidocaldarius is a 393-amino acid protein. Its alignment (http://www.ebi. ac.uk/Tools/msa/clustalw2) with 33 UDP-sulfoquinovose synthases available from the KEGG (http://www.genome. jp/dbgetbin/www_bget?ec:3.13.1.1) and NCBI (http://
blast.ncbi.nlm.nih.gov/Blast.cgi) databases shows a high degree of sequence conservation, with 64 identical amino acids (Fig. 1a).

The phylogenetic analysis using ClustalW2 divides the UDP-sulfoquinovose synthases into four clusters (Fig. 1b). The (cyano)bacterial UDP-sulfoquinovose synthases are distributed in clusters I and II, with UDP-sulfoquinovose synthases from plants mainly localized in cluster IISQD1 from A. thaliana, however, is localized in cluster I. In cluster III, Agl3 of $S$. acidocaldarius and UDP-sulfoquinovose synthases from other Crenarchaeota and hyperthermoacidophilic Euryarchaeota are localized. Apart from them are the UDP-sulfoquinovose synthases from halophilic Euryarchaeota (cluster IV). This cluster is more distant to the other three clusters, showing only 36 identical amino acids with other UDP-sulfoquinovose synthases. The enzyme of the halophilic archaea obviously evolved rather distant to those from plants, hyperthermoacidophilic archaea, and (cyano)bacteria. Among bacteria, the cyanobacterial UDP-sulfoquinovose synthases are predominant, but also other (non-photosynthetic) bacteria contain genes coding for this enzyme. The phylogenetic analysis showed that the occurrence of UDP-sulfoquinovose synthases in all domains of life obviously results from divergent evolution (Fig. 1b). Whether the categorization of the UDP-sulfoquinovose synthases into different clusters also reflects different reaction mechanisms is currently unknown but might be supported by observations made in the course of this work.

Since no crystal structure of Agl3 is available, we chose an approach for the prediction of functional epitopes similar to that used for SQD1 from A. thaliana (Essigmann et al. 1999) before a high-resolution crystal structure of this protein became available (Mulichak et al. 1999). The obtained model clearly shows high similarity of the overall fold of both proteins (for details see Supplemental Information, Figure S1).

Point mutation studies of predicted critical amino acids in $\mathrm{Agl} 3$

Upon comparison of the amino acid sequence of Agl3 with that of other UDP-sulfoquinovose synthases (Fig. 1a) including SQD1 (Fig. 2a) (Mulichak et al. 1999), potential residues of Agl3 were selected that could be involved in catalysis. Very similar to SQD1, our Agl3 model (Fig. 2b) comprises an $\mathrm{N}$-terminal $\mathrm{NAD}^{+}$-binding domain with a highly conserved Rossmann fold and a C-terminal UDPD-glucose binding domain. In SQD1, the catalytic residues were determined to be Thr145, Tyr182 and Lys186, with the latter two being present within the Y-XXX-K motif (Mulichak et al. 1999; Kavanagh et al. 2008). The corresponding amino acids in Agl3 are Thr144, Tyr182 and 
A

Cmaq_1419

Vdis_0939

Ag 13

NP 394533

Bresu 0487

EIO $0 \overline{7} 13$

PFREUD 00050

CLDAP $\overline{1} 4360$

sync $\overline{0} 053$

PMT9312_1757

syc0 945 c

SYNPCC7 $\overline{0} 02$ 2A08 47

Noc 1509

RSP 2569

Rleg 2 3206

Mvan $\overline{5} 143$

CYME_CMR0 12C

PHAT $\bar{R} 21201$

slr $10 \overline{2} 0$

MAE_49250

tl $1 \overline{0} 398$

UCYN 02170

LOC100242408

RCOM 0797290

SQD1 ${ }^{-}$

SELMODRAFT 179226

VOLCADRAFT_109932

Rcas_0045

$\mathrm{SACE}^{-} 5690$

$\mathrm{SqdB}$

Hu ta 1116

NJ 7G 0131

ZOD_14291
S YTMVNNL KS TLNVI YS IREV-NGR IHLLLKMG TLGEF GY P- --A FKLPEDA FVDAV IN KYT I INNL TS TLNVI YA IREV-RRD IH IL KMG TL GEY GY P- --A FK IPEDAFI DAV IQ VYTVI NNE VS TLRVI QA VLEV-DPT IH IL KMG TMGEY GT P- --A FD IPE SI YVEAI VN NE TMVKN ITS TMNL IYA IKDI-VP EA IL KL GTMGE YGTP- --NID IP EG-FFEI EYN LY TVD NN INA TN HL LAA IVES-GLDV HLA HLGTMGVYGYT-TA GLR IP EGYLTVTVQT RY TVD NN ISA TH NLLVA IAAV-SPDT HVV HL GTMGVYGYD- GD GLE IP EGYLDVKVEA RY TVNNNVNA TD NVLCA IVES- GLDI HLV HL GTMGVYGYG- TA GMK IP EGYLDVA VNG RY TVD NNVNG TH NVLCA IVES-GLDI HLVHLGTMGVYGYG-TA GMS IP EGYLTVKVEI RY TVD NNVNG TH NLIAA IVES-GQDI HVV HL GTM GV YG YGS HR GAT IP EG YLKVE VPQ RY TVD NNVNG THNL LAA IVES-NLDI HVVHLGTMGVYGYGS HR GAT IP EG YLKVE VPQ RF TVN NNVNA TH NLLCA CVDV-GL KS HIV HL GTMGVYG YGS HR GAT IP EGYLEVEVVQ RY TVD NNLNG TNNVLCA IVES-GLDI HVV HL GTM GV YGYG-TA GMK IP EGYLDVQVVT RY TVS NNLNA TNNVIAA VVES-GQDI HIAHLGTMGVYGYG-TA GMK IP EGYLQVKVET VY TVNNNVNA THNLLAAMVET-GIDA HLVHLGTMGVYGYS-TV GAP IP EGYIDVSVET NY TVNNNVSA TH NLINALT EL-NLDA HLI HLGTMGVYGYS-TV GAA IP EGYLPVG IET CY TVD NNVNA TH NVLAALVEA-RLDS OLVHL GTMGVYGYE-TS AVD LP EGYLT IS YPD RY TVD NNVNG TMSVLTA VVES-NLDI HVVHLGTMGVYGYG-SS GGK IPEGYLDVLPS RY TVDNNVGG SNNLCCA VI DS-EVDA HI I HL GTMGVYGYG-TS GGE IP EGYIDVT LPG VL TQANNVLGNL NL LYALKED- FP DC HLVKLGTMGE YGTP---NID IE EGYIT IE HKG VL TQVNNVVGNL NI LYAMKEE- FP EA HLVKL GTMGE YGTP---NID IE EGYIT IE HNG VF TQVNNVVG TLNLLYA IH TH-NPDC HLVKLGTMGE YGTP- --NID IE EGYIT IE HNG VF TQVNNVVG TL NI LYC IKEY-FPDCHLI KL GTMGE YGTP- --NID IE EG YIT IE HNG VF TQHNNVIGTLNVLFA IKEF-RE QCHLVKLGTMGE YGTP---NID IE EGYIT IT HNG VF TQHNNVIG TL NVLFA IKEF-RE EC HLVKLGTMGE YGTP- --NID IE EGYIT IT HNG VY TQHNNVIG TL NVLFA IKEF-GE ECHLVKLGTMGE YGTP- --NID IE EGYIT IT HNG VY TQHNN IVGTI NVLFA IKEF-SPECHLVKLGTMGE YGTP- --NID IE EGFIT IT HNG VF TQT NNVVG TL NVLFA IKEL-KPDCHLVKLGTMGE YGTP- --NID IE EGYLTVT HNG VF THVNNVVG TL NLIYALADH-APDC HLVKLGTMGE YGTP- --NID IE EGYIT IT HKG VY TQQNNVVGNL NVLFA IQEV-DPDI HLVKLGTMGE YGTP---NID IE EGWLEVT HKG NY TQQNNMQA TRNL VFGLHEND LVDT HLI ET TTT GVYGAP- --EFP IP EGGTVME HQD NY TQH NNMOA TRNL LWGLE EHD LT DT HFI ET TTT GVY GAP- --EFP IP EGGATME NQG NF TQH NN LQA TRNL LWS LE EHGLE ET HFI ET TTT GV Y GAP- --EFP IP EGGATME HRG NY TQHNNIQS TRNL LWGLE ENGLTDT HFI ET TIT GVY GAP- -- EFP IP EG GATME NGG

B Cluster IV

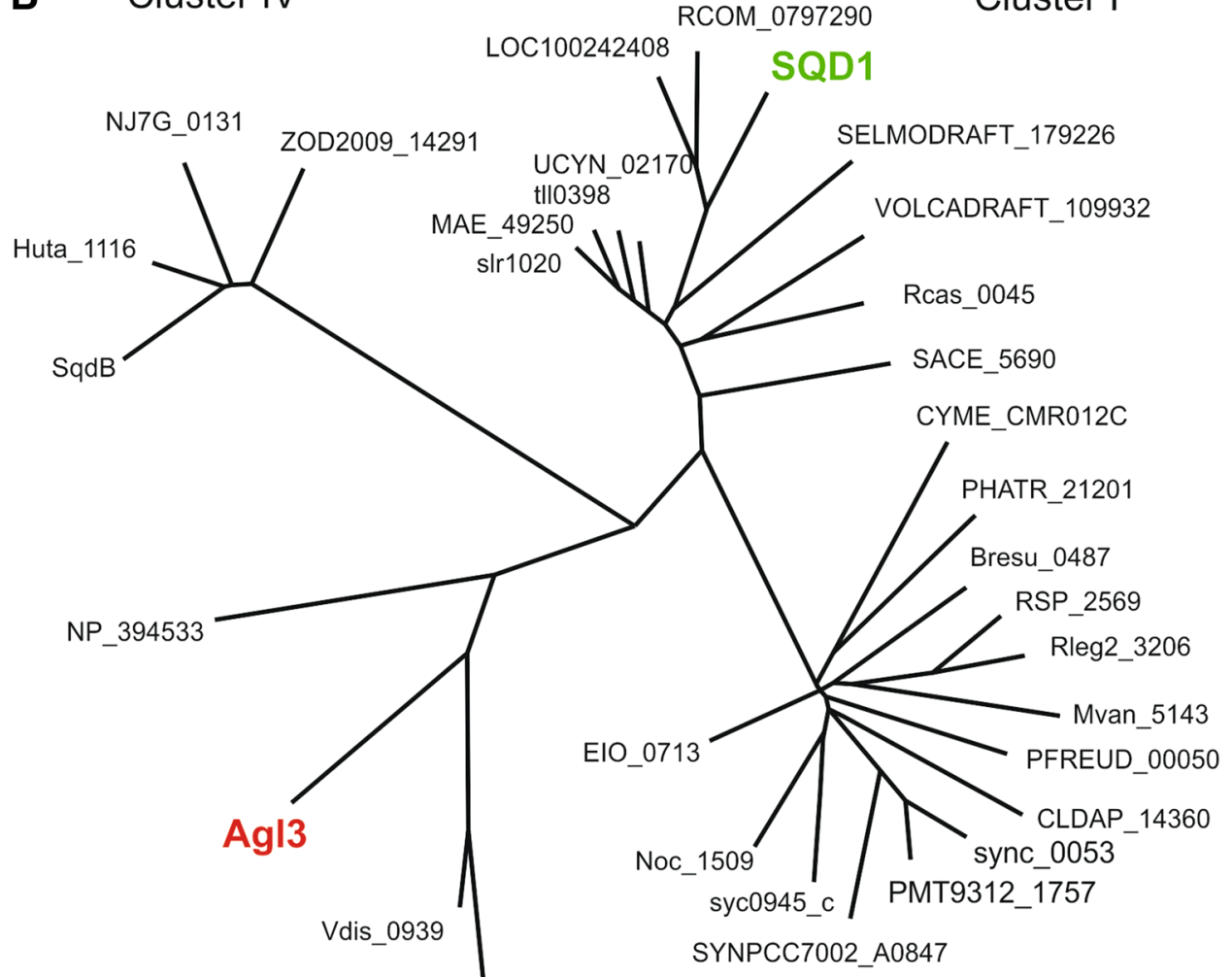

Cluster III

Cmaq_1419

Cluster II 
४Fig. 1 Homology alignment of amino acid sequences of UDPsulfoquinovose synthases from plants, green algae, (cyano) bacteria, and archaea. a Alignment of selected regions of UDPsulfoquinovose synthases showing the identical amino acids (colored in red) from S. acidocaldarius, plants, (cyano)bacteria, and selected species from thermophilic and extreme halophilic archaea. b Phylogenetic relationship of UDP-sulfoquinovose synthases from archaea, (cyano)bacteria, and plants. The designation of organisms and genes used for the construction of the phylogenetic tree is (in alphabetical order): Arabidopsis thaliana, SQD1; Brevundimonas subvibrioides, Bresu_0487; Caldilinea aerophila, CLDAP_14360; Caldivirga maquilingensis, Cmaq_1419; Cyanidioschyzon merolae strain 10D, CYME_CMR012C; Cyanobacterium UCYNA, UCYN_02170; Haladaptatus paucihalophilus, ZOD2009_14291; Haloquadratum walsbyi, SqdB; Halorhabdus utahensis, Huta_1116; Ketogulonicigenium vulgare, EIO_0713; Microcystis aeruginosa NIES-843, MAE_49250; Mycobacterium vanbaalenii, Mvan_5143; Natrinema sp. J7-2, NJ7G_0131; Nitrosococcus oceani ATCC 19707, Noc_1509; Phaeodactylum tricornutum CCAP 1055/1, PHATR_21201; Propionibacterium freudenreichii, PFREUD_00050; Rhizobium leguminosarum bv. trifolii, Rleg2_3206; Rhodobacter sphaeroides, RSP_2569; Ricinus communis, RCOM_0797290; Roseiflexus castenholzii, Rcas_0045; Saccharopolyspora erythraea, SACE_5690; Selaginella moellendorffii, SELMODRAFT_179226; Sulfolobus acidocaldarius, Agl3; Synechococcus sp., sync_0053; Synechococcus sp. PCC 7002, SYNPCC7002_A0847; Synechocystis sp. PCC 6803, slr1020; Thermoplasma acidophilum, NP_394533; Thermosynechococcus elongatus, tll0398; Vitis vinifera, LOC100242408; Volvox carteri f. nagariensis, VOLCADRAFT_109932; Vulcanisaeta distributa, Vdis_0939
Lys186. From the respective alanine mutations (Tables 1, 2) the mutant T144A fully retained activity, whereas Ala replacement of His95, Arg101, Met145, Tyr182 and Lys186 (Fig. 2b) in Agl3 rendered the enzyme inactive. Both S180A and T185A mutant proteins showed impaired conversion of UDP-D-glucose to UDP-D-glucose-5,6-ene (Tables 1, 2).

Since most of the selected mutants were inactive and did not allow any prediction of a possible reaction pathway, additional mutations were introduced which open the possibility of a dehydratase mechanism for Agl3 (Gross et al. 2001; Hegeman et al. 2001). It was previously shown that in the dehydration reaction of dTDPD-glucose-4,6-dehydratase (Allard et al. 2002) and GDPD-mannose-4,6-dehydratase (Somoza et al. 2000) the two active site amino acids tyrosine and glutamic acid are crucial for the acid-catalyzed release of the hydroxyl group at C-6 of the substrate. According to our active site model of Agl3 (Fig. 2b), these amino acids could be represented by Tyr148 and Glu147 in Agl3, supporting a possible UDPD-glucose dehydratase activity for UDP-sulfoquinovose biosynthesis. The respective alanine mutants (Tables 1, 2) showed strongly impaired conversion of UDP-D-glucose to UDP-D-glucose-5,6-ene (Fig. 2c) in the absence
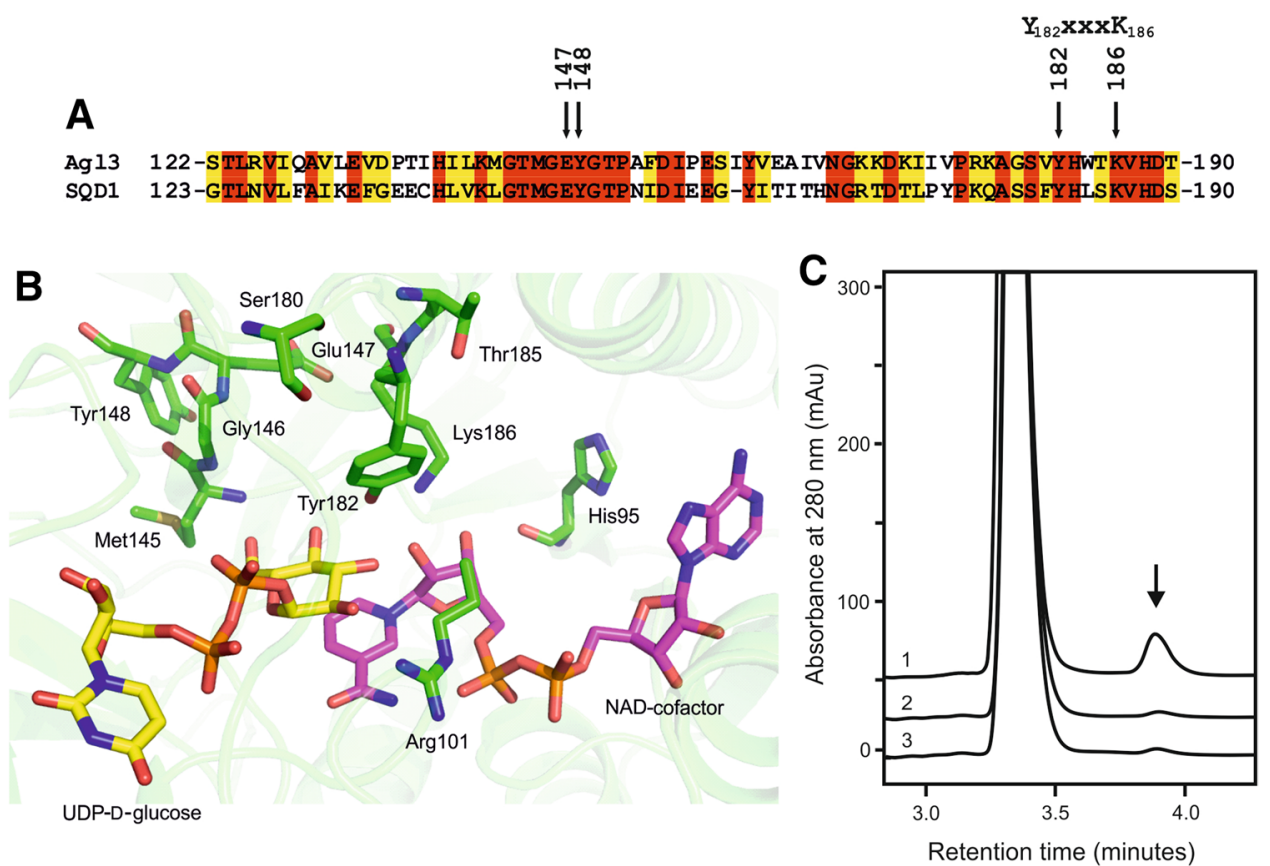

Fig. 2 Partial amino acid comparison, close-up of the active site, and reactivity of wild-type and specific mutants of Agl3. a Selected region of the sequence alignment of Agl3 and SQD1 demonstrating a high degree of amino acid similarity of both enzymes. The positions of E147, Y148, and the characteristic Y-XXX-K motif of SDR proteins are indicated (Kavanagh et al. 2008). b Homology model of the active site of Agl3 with the critical amino acids drawn in green sticks, the NAD-cofactor in pink and the substrate in yellow. For clarity the backbone of residues 96-108 is not shown; (the figure was generated by pymol version 1.2r1). $\mathbf{c}$ In vitro conversion of UDP-D-glucose to UDP-D-glucose-5,6-ene (arrow) in absence of sulfite by the wild-type Agl3 (graph 1), Y148A mutant (graph 2), and E147A mutant (graph 3) 
Table 1 Alanine mutations of selected amino acids of Agl3: used primers, primer sequence, target mutation and overall mutant enzyme activity

\begin{tabular}{|c|c|c|c|}
\hline Primer name & Primer sequence & Target mutation & Mutant enzyme activity $^{\mathrm{a}}$ \\
\hline Sqsyn1-Forward-H95A & 3'-GCCATAGTGGCTTTCGCTGAG-5' & Histidine 95 & \\
\hline Sqsyn2-Reverse-H95A & 3'-CTCAGCGAAAGCCACTATGGC-5' & Histidine 95 & - \\
\hline Sqsyn3-Forward-R101A & 3'-GCTGAGCAGGCTTCTGCTCCG-5' & Arginine 101 & \\
\hline Sqsyn4-Reverse-R101A & 3'-CGGAGCAGAAGCCTGCTCAGC-5' & Arginine 101 & - \\
\hline Sqsyn5-Forward-T144A & 3'-AAGATGGGTGCTATGGGTGAG-5' & Threonine 144 & \\
\hline Sqsyn6-Reverse-T144A & 3'-CTCACCCATAGCACCCATCTT-5' & Threonine 144 & ++ \\
\hline Sqsyn7-Forward-M145A & 3'-ATGGGTACCGCTGGTGAGTAT-5' & Methionine 145 & \\
\hline Sqsyn8-Reverse-M145A & 3'-ATACTCACCAGCGGTACCCAT-5' & Methionine 145 & - \\
\hline Sqsyn19-Forward-E147A & 3'-ACCATGGGTGCCTATGGAACACCT-5' & Glutamic acid 147 & \\
\hline Sqsyn20-Reverse-E147A & 3'-AGGTGTTCCATAGGCACCCATGGT-5' & Glutamic acid 147 & $( \pm)$ \\
\hline Sqsyn21-Forward-Y148A & 3'-CATGGGTGAGGCTGGAACACCT-5' & Tyrosine 148 & \\
\hline Sqsyn22-Reverse-Y148A & 3'-AGGTGTTCCAGCCTCACCCATG-5' & Tyrosine 148 & $( \pm)$ \\
\hline Sqsyn9-Forward-S180A & 3'-GCGGGTGCTGTTTATCAC-5' & Serine 180 & \\
\hline Sqsyn10-Reverse-S180A & 3'-GTGATAAACAGCACCCGC-5' & Serine 180 & $(+)$ \\
\hline Sqsyn11-Forward-Y182A & 3'-GGTTCTGTTGCTCACTGGACT-5' & Tyrosine 182 & \\
\hline Sqsyn12-Reverse-Y182A & 3'-AGTCCAGTGAGCAACAGAACC-5' & Tyrosine 182 & - \\
\hline Sqsyn15-Forward-T185A & 3'-TATCACTGGGCTAAGGTTCAT-5' & Threonine 185 & \\
\hline Sqsyn16-Reverse-T185A & 3'-ATGAACCTTAGCCCAGTGATA-5' & Threonine 185 & $(+)$ \\
\hline Sqsyn17-Forward-K186A & 3'-CACTGGACTGCTGTTCATGAT-5' & Lysine 186 & \\
\hline Sqsyn18-Reverse-K186A & 3'-ATCATGAACAGCAGTCCAGTG-5' & Lysine 186 & - \\
\hline
\end{tabular}

${ }^{a}$ Qualitative determination: ++ strongly active, + active, $(+)$ lesser active, $( \pm)$ less active, - inactive protein

Table 2 Alanine mutations of selected amino acids of Ag13: enzymatic characterization of wild-type Agl3 and the mutants E147A and Y148A

\begin{tabular}{llllll}
\hline Enzyme protein & Total reaction volume $(\mu \mathrm{l})$ & Amount of enzyme $(\mathrm{mg})$ & Total yield $\left(\mathrm{Units}^{\mathrm{a}}\right)$ & $\begin{array}{l}\text { Specific activity }\left(\mathrm{U} \mathrm{mg}^{-1} \text { of }\right. \\
\text { protein })\end{array}$ & Total activity $(\%)$ \\
\hline Wild-type Ag13 & 200 & 0.121 & 0.00673 & 0.0556 & 100 \\
E147A mutant & 200 & 0.135 & 0.00094 & 0.0069 & 12.4 \\
Y148A & 200 & 0.129 & 0.00109 & 0.0084 & 15 \\
\hline
\end{tabular}

${ }^{a}$ One unit is defined as micromole of product (UDP-D-glucose-5,6-ene) produced per milligrams of enzyme protein in $30 \mathrm{~min}$

of sulfite, supporting their important roles in the catalytic activity of Agl3 and the assumed dehydratase-like function. To ensure that the observed decrease in activity was not a result of protein instability, far-UV circular dichroism spectra of wild-type Agl3 and the variants E147A and Y148A were recorded. Figure 3 demonstrates the mainly $\alpha$-helical structure of Agl3 as well as the almost identical overall secondary structure composition of the three mutant proteins.

In the homology model of the putative active site of Agl3, the residues Glu147 and Tyr148 are at a distance of $>5 \AA$ away from UDP-D-glucose (Fig. 2b). This suggests that either a conformational change of the G-XX-G-XX-G motif must occur to facilitate the reaction or UDP-D-glucose needs to change its orientation to favorably interact with these residues. Overall, UDP-sulfoquinovose synthases contain several highly conserved glycine and proline residues, suggesting a highly conserved structural mode of switching Agl3 from an inactive to an active conformation.

Catalytic conversion of UDP-D-glucose

to UDP-D-glucose-5,6-ene in the absence of a sulfur donor

Previously, we demonstrated that recombinant Agl3 from $S$. acidocaldarius is active in vitro, converting UDP-D-glucose and sulfite to UDP-sulfoquinovose in a yield of approximately $10 \%$ (Meyer et al. 2011). Here, we show that in the absence of sulfite, the in vitro conversion of UDPD-glucose results in the formation of UDP-D-glucose-5,6ene (Fig. 4). This new reaction product eluted at a retention time of $3.8 \mathrm{~min}$ in the RP-HPLC experiment (Fig. 4a). The Agl3-catalyzed conversion of UDP-D-glucose was further investigated by NMR spectroscopy and ESI-MS (Fig. 4b, c). Supplementation of the reaction mixture with $\mathrm{NAD}^{+}$, 
Fig. 3 Far-UV CD spectra of $7 \mu \mathrm{M}$ wild-type $\mathrm{Agl} 3$ and the mutants E147A and Y148A in $20 \mathrm{mM}$ phosphate buffer, $\mathrm{pH}$ 7.0

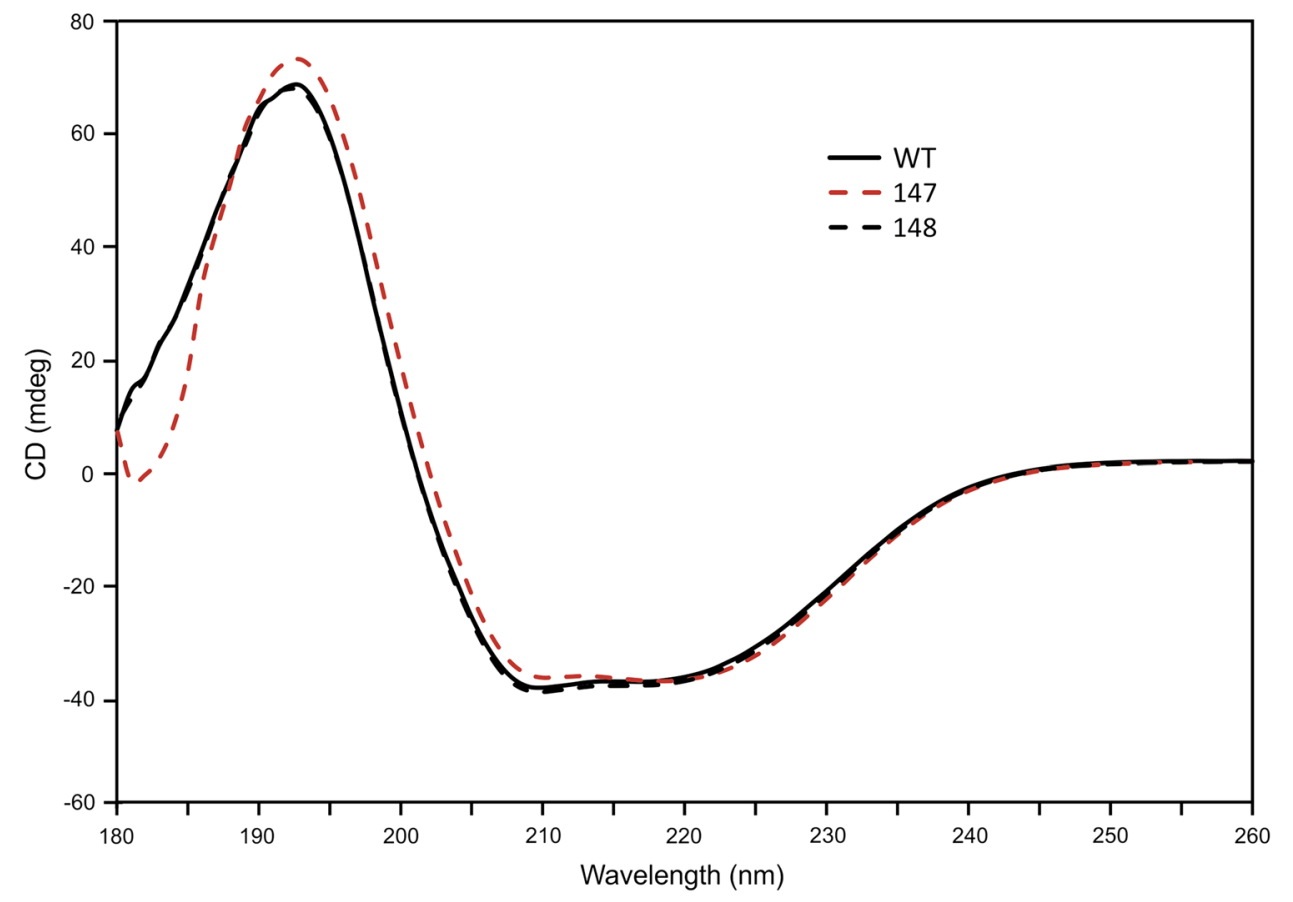

NADH or FAD to a final concentration of $1 \mathrm{mM}$, each, did not result in a higher catalytic conversion of UDP-D-glucose, as was concluded from the yield and retention time of the reaction product upon RP-HPLC separation (not shown). The ${ }^{1} \mathrm{H}$ NMR spectrum of that sample revealed the signals of non-reacted UDP-D-glucose (verified by using an UDP-D-glucose standard) and of a second compound in a ratio of 2.8:1, as was deduced from the integrated signals of the respective anomeric protons (at 5.5 and $5.6 \mathrm{ppm}$, respectively). The minor, slightly low-field shifted anomeric proton of the second compound displayed a heteronuclear coupling to ${ }^{31} \mathrm{P}$, and the presence of an intact diphosphate unit was confirmed by the chemical shifts in the ${ }^{31} \mathrm{P}$ NMR spectrum (Fig. 4b, inset). Despite overlapping signals from the UDP-component of UDP-D-glucose and the reaction product, the remaining signals could be fully assigned using COSY, TOCSY, edHSQC and HMBC data. Thus, structural proof for the presence of an exocyclic double bond was obtained from the edited HSQC spectrum (Fig. 4b). Two low-field shifted ${ }^{1} \mathrm{H}$ NMR signals were observed at 4.81 and $4.78 \mathrm{ppm}$ with a correlation to a ${ }^{13} \mathrm{C}$ NMR signal at $98.5 \mathrm{ppm}$. Both protons displayed small values for the geminal coupling constant as well as allylic spin-spin couplings to $\mathrm{H}-4^{\prime \prime}$. The latter signal revealed a large coupling constant confirming a trans-orientation relative to $\mathrm{H}-3^{\prime \prime}$, in agreement with a gluco-configuration. Final support for the proposed UDP-D-glucose-5,6-ene structure was derived from HMBC-correlations of $\mathrm{H}-4$ " as well as $\mathrm{H}-1$ " to a ${ }^{13} \mathrm{C}$ NMR signal at $155.3 \mathrm{ppm}$, which are in full agreement with the partial structure of an exocyclic enol ether (Table 3). Based on these data, the structure of the enzymatic reaction product could be unambiguously determined as UDP 6-deoxy-D-xylo-hex-5-enose (UDP-Dglucose-5,6-ene). Signals for keto or hydrated keto groups as well as signals for deuterated UDP-D-glucose in the product mixture could not be detected within the detection limit of the NMR instrument. Also, in situ monitoring of the enzymatic reaction in an NMR tube for $5 \mathrm{~h}$ at $333 \mathrm{~K}$ $\left(60{ }^{\circ} \mathrm{C}\right)$ revealed a slow formation of the UDP-D-glucose5,6-ene product but did not provide direct evidence of a 4-keto intermediate ( $\mathrm{Li}$ et al. 2014).

The MS-spectrum of the reaction mixture of UDPD-glucose conversion showed a major peak at $565.0 \mathrm{u}$ (corresponding to the substrate), accompanied by two smaller peaks at 566.0 and $567.0 \mathrm{u}$, respectively, and one further, though even smaller, peak at $547.0 \mathrm{u}$ showing the same pattern of accompanying peaks, each one unit apart, as seen with the major peak, but with much less intensity (Fig. 4c).

Formation of UDP-D-glucose-5,6-ene in the course of UDP-sulfoquinovose biosynthesis

To investigate the formation of UDP-D-glucose-5,6-ene (Fig. 5) in detail, the RP-HPLC profile of the conversion of $3 \mathrm{mM}$ UDP-D-glucose to UDP-sulfoquinovose was compared at high (3 mM; Fig. 5a, graph 1), and low sulfite concentration ( $0.1 \mathrm{mM}$; Fig. $5 \mathrm{a}$, graph 2). At $3 \mathrm{mM}$ sulfite, Agl3 rapidly converted UDP-D-glucose to UDP-sulfoquinovose (Fig. 5a, graph 1), with complete product formation as indicated by the lack of detectable UDP-D-glucose-5,6ene. In contrast, at $0.1 \mathrm{mM}$ sulfite, a considerable accumulation of UDP-D-glucose-5,6-ene occurred in the reaction 
Fig. 4 Conversion of UDPD-glucose by Agl3 in the absence of sulfite (a) RP-HPLC analysis of the conversion of UDP-D-glucose to the UDPD-glucose-5,6-ene intermediate (arrow at $3.8 \mathrm{~min}$ ) in the presence of Agl3 (graph 1). In the negative control experiment (absence of Agl3; graph 2), no conversion was observed. b NMR expansion plot of an edHSQC spectrum of the mixture of UDP-D-glucose (major component) and UDP-D-glucose5,6-ene (minor component from the enzymatic conversion). The inset (b) shows an expansion plot of the ${ }^{31} \mathrm{P}$ NMR spectrum of the mixture displaying the intact diphosphate linkages of both compounds. $\mathbf{c}$ ESI-MS analysis of UDP-D-glucose5,6-ene
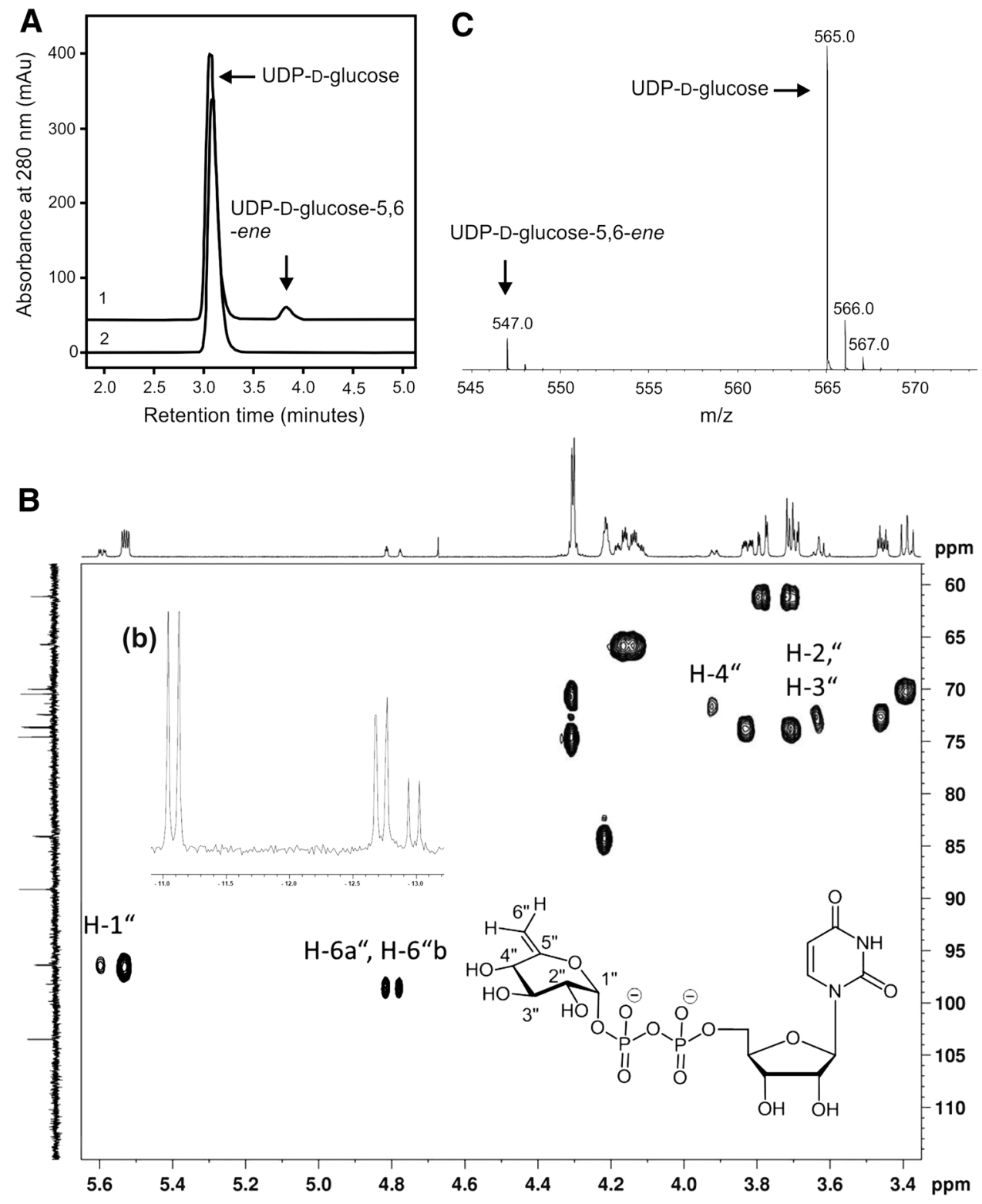

mixture (Fig. 5b, graph 2). Under sulfite-saturated conditions, UDP-D-glucose is first converted to UDP-D-glucose5,6-ene, followed by conversion into UDP-sulfoquinovose (Fig. 5c). Based on the kinetics of UDP-sulfoquinovose biosynthesis, in combination with the accumulation of UDP-D-glucose-5,6-ene in the absence of sulfite, we conclude that conversion of UDP-D-glucose to UDP-sulfoquinovose by $\mathrm{Agl} 3$ is a two-step reaction with UDP-D-glucose-5,6-ene being a crucial reaction intermediate of this pathway (Figs. 4, 5). However, due to the available analytical tools in our laboratory, we were only able to isolate and unambiguously characterize starting and end points of these reactions. The appearance of UDP-D-glucose-5,6-ene is taken as an additional indication of a dehydration step being included in the complex Agl3 reaction mechanism.
Preliminary investigations of the dehydration mechanism of $\mathrm{Agl} 3$ and role of the NAD-cofactor

Monitoring of the conversion of UDP-D-glucose is challenging, because of the low in vitro activity of Agl3 and the fact that the absorption spectra of UDP-D-glucose, UDP-D-glucose-5,6-ene and UDP-sulfoquinovose are identical. Supplementing the reaction mixture with $\mathrm{NAD}^{+}$during Agl3 activity measurement did not lead to accumulation of NADH (monitored at $340 \mathrm{~nm}$ ). This is consistent with our observation that supplementing with $\mathrm{NAD}^{+}$does not increase the catalytic activity of Agl3 (data not shown) and indicative of a tight binding of $\mathrm{NAD}^{+}$to the polypeptide matrix of $\mathrm{Agl} 3$ and no exchange with the medium. 
Table 3 NMR data of UDP-D-glucose-5,6-ene

\begin{tabular}{lllll}
\hline Atom & ${ }^{1} \mathrm{H}(\mathrm{ppm})$ & $J(\mathrm{~Hz})$ & ${ }^{13} \mathrm{C}(\mathrm{ppm})$ & HMBC correlation \\
\hline $1^{\prime \prime}$ & 5.59 & $3.0 / 7.9$ & 96.4 & 155.3 \\
$2^{\prime \prime}$ & 3.63 & n.d. & 72.1 & - \\
$3^{\prime \prime}$ & 3.62 & $9.7 / 9.4$ & 72.7 & - \\
$4^{\prime \prime}$ & 3.91 & $9.4 / 2 \times 2.0$ & 71.6 & - \\
$5^{\prime \prime}$ & - & - & 155.3 & - \\
$6^{\prime \prime} \mathrm{a}$ & 4.81 & $1.7 / 1.9$ & 98.5 & 155.3 \\
$6^{\prime \prime} \mathrm{b}$ & 4.78 & $1.6 / 2.1$ & 98.5 & - \\
$1^{\prime}$ & 5.90 & n.d. & 89.3 & - \\
$2^{\prime}$ & $4.31-4.29$ & n.d. & 74.6 & - \\
$3^{\prime}$ & $4.31-4.29$ & n.d. & 70.7 & - \\
$4^{\prime}$ & $4.22-4.20$ & n.d. & 84.3 & - \\
5a'/5'b & $4.18-4.11$ & n.d. & 65.8 & - \\
2 & - & - & n.d. & - \\
4 & - & - & n.d. & - \\
5 & 5.90 & - & 103.7 & - \\
6 & 7.88 & 8.1 & 142.9 & - \\
& $31 \mathrm{P}(\mathrm{ppm})$ & & & - \\
$5^{\prime}$-linked & -11.08 & 20.5 & - & - \\
$1^{\prime \prime}$-linked & -12.98 & 20.5 & - & - \\
\hline
\end{tabular}

n.d. not determined

The nature of the tightly bound prosthetic group in Agl3 was investigated after chloroform extraction (Fig. 6a). RPHPLC analysis confirmed the presence of $\mathrm{NAD}^{+}$in $\mathrm{Agl} 3$ by comparison with authentic $\mathrm{NAD}^{+}$reference material (Fig. 6a, graph 2). Fractionation and spectrophotometric analysis of the extracted cofactor showed a typical $\mathrm{NAD}^{+}$ absorption spectrum in the oxidized state (Fig. 6b, spectrum 1). Subsequently, the nature of the extracted NADcofactor was confirmed as $\mathrm{NAD}^{+}$in an in vitro biocatalytic experiment (Fig. 6b, spectra 2 and 3 ) by adding trace amounts of NAD(P)-dependent glucose dehydrogenase and glucose (1 mM final concentration) to the extracted cofactor. After 2 min of incubation at room temperature, $\mathrm{NAD}^{+}$ was reduced, evidenced by an increase of absorbance (Fig. 6b, spectrum 2), which was even further pronounced after $3 \mathrm{~min}$ of incubation (Fig. 6b, spectrum 3), since glucose dehydrogenase converted glucose to D-glucono1,5-lactone thereby transferring the electrons to $\mathrm{NAD}^{+}$and yielding NADH.

Agl3 was, in any case, tested as a holo enzyme because all attempts to prepare NAD-free apo-Agl3 failed. Extensive dialysis ( 2 days) did not remove the bound NADcofactor; the absorption spectrum of Agl3 at $340 \mathrm{~nm}$ still showed the presence of cofactor inside the enzyme. Dialysis for longer time led to precipitation of $\mathrm{Agl} 3$ and complete loss of activity (not shown).

Since determination of the NAD-dependent activity of Agl3 could not be monitored at steady-state conditions, the pre-steady-state kinetics of the reaction was investigated by stopped-flow experiments. We probed whether the bound cofactor is able to accept electrons from UDP-D-glucose at $65{ }^{\circ} \mathrm{C}$, mimicking the natural growth temperature of $S$. acidocaldarius. As depicted in Fig. 6c (spectrum 1), in the resting state of recombinant $\operatorname{Ag} 13(2 \mu \mathrm{M})$, the spectrum of the pyridine nucleotide cofactor suggests the occurrence of a mixture of $\mathrm{NAD}^{+}$and $\mathrm{NADH}$. Upon addition of
Fig. 5 HPLC analysis of the conversion of UDP-D-glucose and sulfite to UDP-sulfoquinovose by Agl3 (a) UDP-sulfoquinovose biosynthesis was analyzed under sulfite-saturated (graph 1) and unsaturated conditions ( $\operatorname{raph} 2$ ). b The UDPD-glucose-5,6-ene is present at sulfite-saturated UDP-sulfoquinovose biosynthesis (graph 1; only trace amounts), and under unsaturated conditions ( graph 2). c dehydration of UDP-D-glucose is a key catalytic activity of Agl3
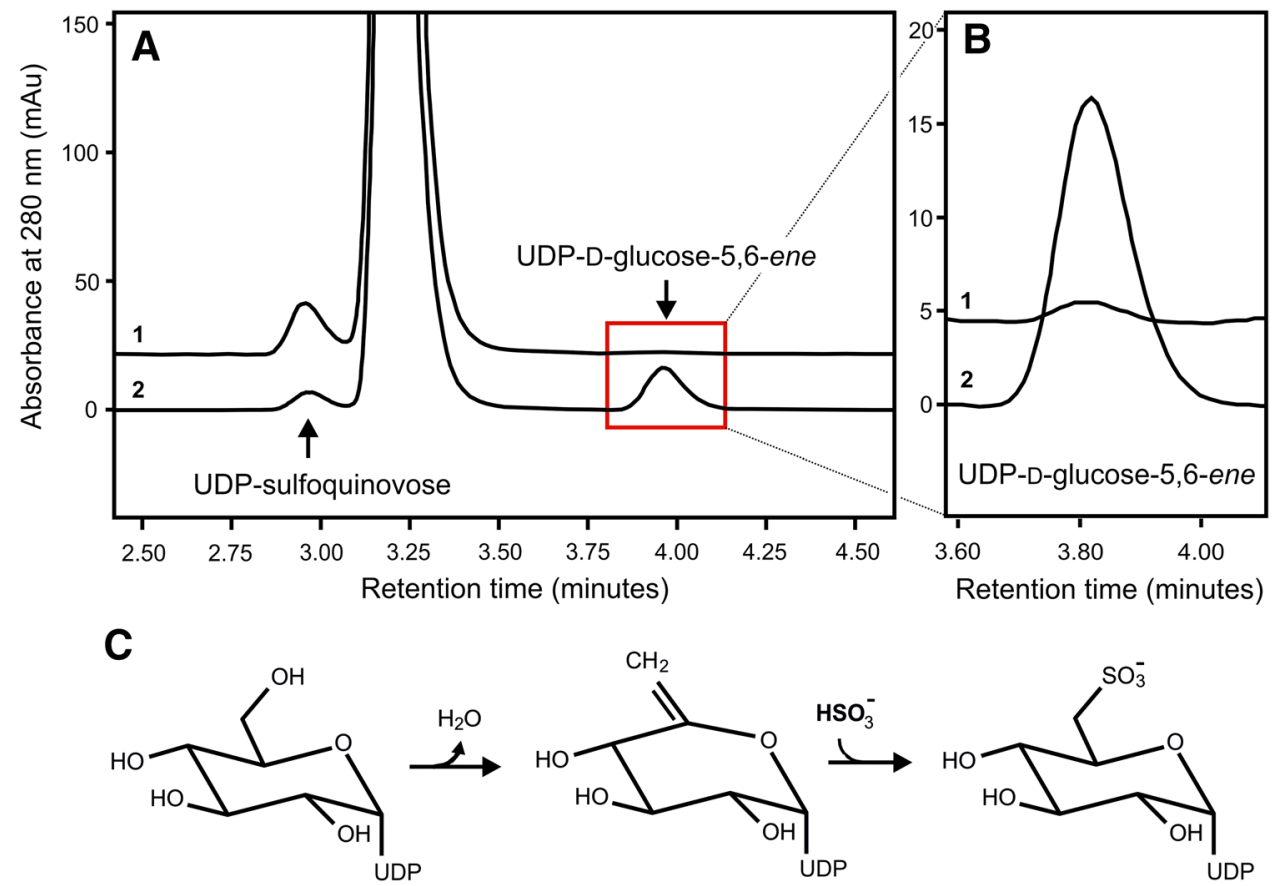
A
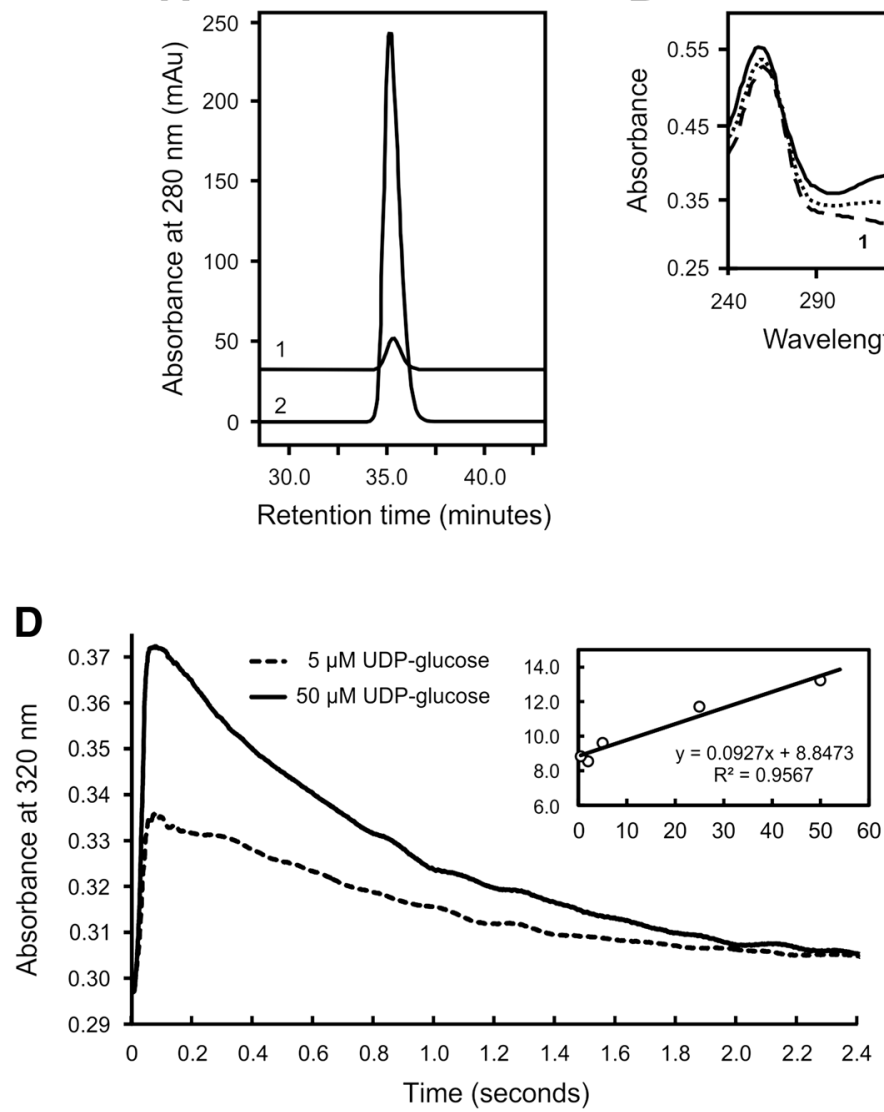

B

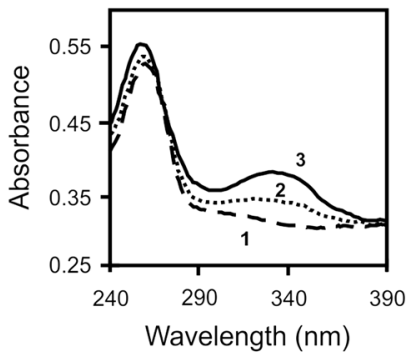

C

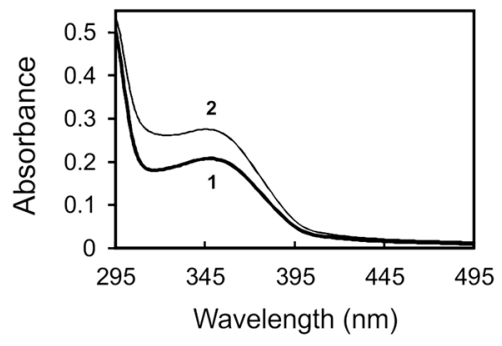

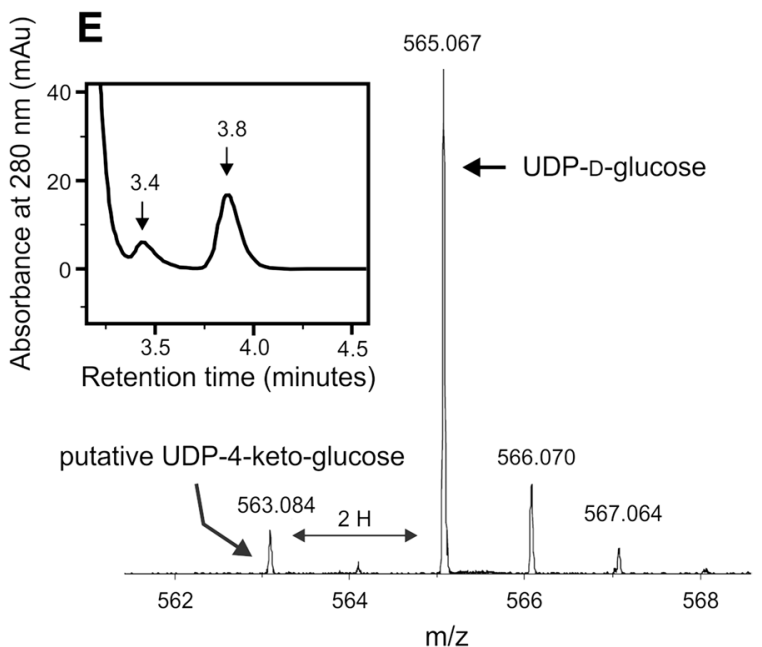

Fig. 6 Cofactor analysis and kinetics of $\mathrm{NAD}^{+}$reduction of UDPD-glucose. a RP-HPLC analysis of $\mathrm{NAD}^{+}$extracted from Agl3 in oxidized form (graph 1) compared to $\mathrm{NAD}^{+}$reference (graph 2). b Absorption spectra of the extracted cofactor in the fully oxidized $\left(\mathrm{NAD}^{+}\right.$; spectrum 1) and reduced (NADH) state. Reduction was mediated by glucose dehydrogenase and glucose. Spectra were recorded after 2 min (spectrum 2) and 5 min (spectrum 3). c Absorption spectrum of Agl3 in the resting state (spectrum 1) suggesting a mixed oxidation state of the tightly bound cofactor. Spectrum 2 is formed immediately after addition of $50 \mu \mathrm{M}$ UDP-D-glucose. d Stopped-flow analysis of the reduction of the bound NAD-cofactor

$50 \mu \mathrm{M}$ UDP-D-glucose there was a rapid formation of fully reduced NADH (Fig. 6c, spectrum 2) followed by a slow re-oxidation to the mixed $\mathrm{NAD}^{+} / \mathrm{NADH}$ state. The corresponding time traces of this biphasic reaction are shown in Fig. $6 \mathrm{~d}$ for 5 and $50 \mu \mathrm{M}$ UDP-D-glucose, respectively. The first rapid absorbance increase at $320 \mathrm{~nm}$ is dependent on the concentration of the electron donor. Upon fitting this phase by a single-exponential function, $k_{\mathrm{obs}}$ values could be estimated. From the slope of the linear plot of $k_{\text {obs }}$ values versus the concentration of UDP-D-glucose, an apparent bimolecular rate constant of $\sim 9.3 \times 10^{4} \mathrm{M}^{-1} \mathrm{~s}^{-1}$ could be estimated at $65{ }^{\circ} \mathrm{C}$ and $\mathrm{pH}$ 7.0. The high intercept of $8.8 \mathrm{~s}^{-1}$ suggests that transiently formed NADH becomes re-oxidized. After about $3 \mathrm{~s}$ the mixed $\mathrm{NAD}^{+} / \mathrm{NADH}$ state of the enzyme sample was established again (Fig. 6d). The by UDP-D-glucose. Two representative time traces for the reaction between $2 \mu \mathrm{M} \mathrm{Agl3}$ and 5 or $50 \mu \mathrm{M}$ UDP-D-glucose are depicted. The first rapid increase at $320 \mathrm{~nm}$ depends on the UDP-D-glucose concentration. The inset shows the corresponding plot of the $k_{\mathrm{obs}}$ values for this reaction versus the concentration of the electron donor. e RP-HPLC analysis and MS analysis of a new reaction product at 3.4 min retention time (HPLC graph) and a molecular mass of 563 gram $/ \mathrm{mole}$ (mass spectrum), extracted during the conversion of UDPD-glucose to UDP-D-glucose-5,6-ene by chloroform in the absence of sulfite and at an $\mathrm{Agl} 3$ concentration of $2.7 \mathrm{mg} \mathrm{ml}^{-1}$

second slower phase (i.e., absorbance decrease at $320 \mathrm{~nm}$ ) did not depend on the sugar concentration. Consistent with the literature (Mulichak et al. 1999, 2002; Hegeman et al. 2001), we propose that the conversion of UDP-D-glucose to UDP-D-glucose-5,6-ene by Agl3 is initiated by oxidation of the hydroxyl group at C-4 of the substrate by $\mathrm{NAD}^{+}$, leading to NADH. This is first demonstration of the rapid electron transfer between UDP-D-glucose and $\mathrm{NAD}^{+}$in a UDP-sulfoquinovose synthase (Fig. 6c, d).

The oxidation of UDP-D-glucose was accomplished at high enzyme concentration $\left(2.7 \mathrm{mg} \mathrm{ml}^{-1}\right)$. Chloroform was directly added to the reaction mixture, resulting in an instant unfolding of Agl3 and release of the reaction product into the water phase. RP-HPLC analysis of the latter showed the presence of the new compound at $3.4 \mathrm{~min}$ 
Fig. 7 Conversion of UDPD-glucose to UDP-D-glucose5,6-ene by Agl3 at an excess of deuterium. a Conversion of UDP-D-glucose to UDP-Dglucose-5,6-ene reaching an equilibrium reaction at steadystate level. b MS analysis of the conversion of UDP-D-glucose to UDP-D-glucose-5,6-ene in $\mathrm{H}_{2} \mathrm{O}$-phosphate buffer compared to (c) its conversion in $\mathrm{D}_{2} \mathrm{O}$ phosphate buffer. d Natural isotope distribution pattern of ESI-MS in the negative mode in $\mathrm{H}_{2} \mathrm{O}$-phosphate UDP-D-glucose measured by
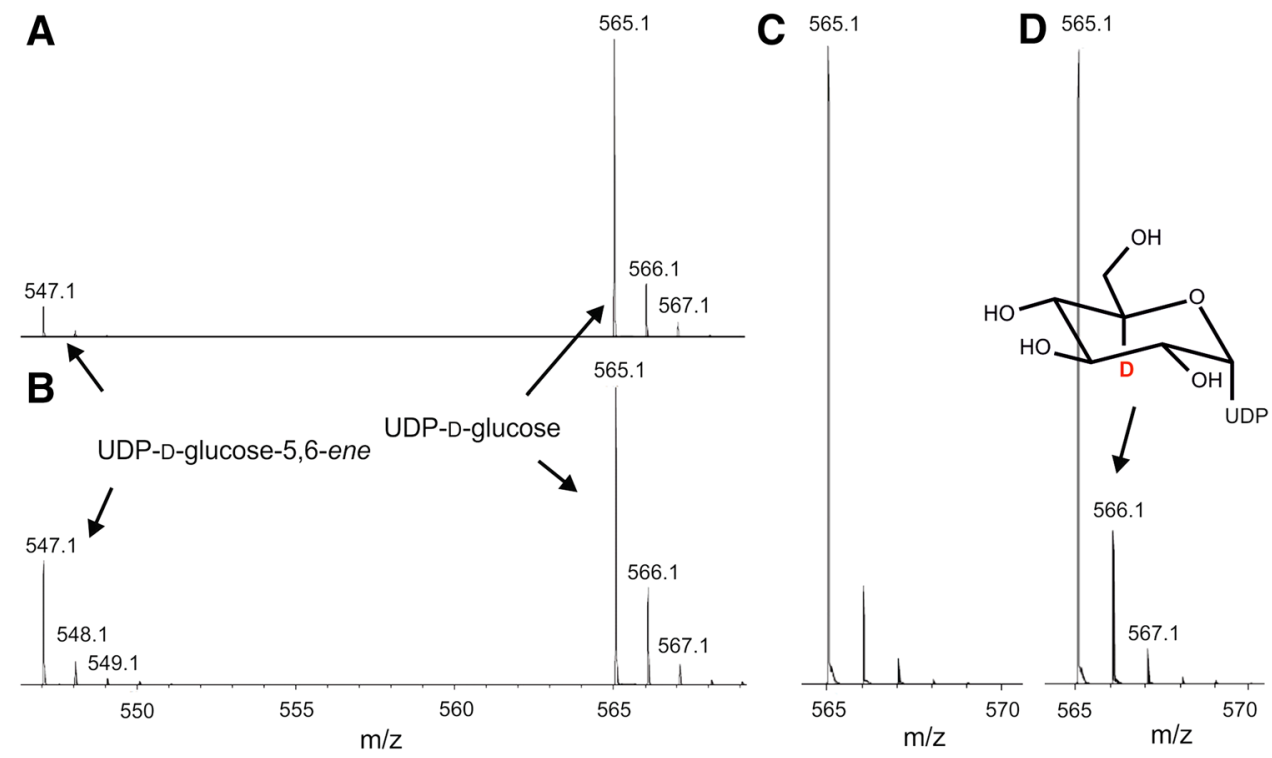

Table 4 Ratio of isotope distribution of UDP-D-glucose and UDP-D-glucose-5,6-ene from enzymatic conversion performed in $\mathrm{H}_{2} \mathrm{O}$ and excess of $\mathrm{D}_{2} \mathrm{O}$

\begin{tabular}{lll}
\hline$m / z$ & $\begin{array}{l}\text { Ratio of isotope distribution of UDP-D-glucose-5,6-ene } \\
\text { from enzymatic conversion in excess } \mathrm{D}_{2} \mathrm{O}\end{array}$ & $\begin{array}{l}\text { Ratio of isotope distribution of } \\
\text { UDP-D-glucose-5,6-ene from } \\
\text { enzymatic conversion in } \mathrm{H}_{2} \mathrm{O}\end{array}$ \\
\hline 547 & 1 & 1 \\
548 & 0.185 & 0.195 \\
549 & 0.053 & 0.053 \\
\hline$m / z$ & Ratio of isotope distribution of UDP-D-glucose from & $\begin{array}{l}\text { Ratio of isotope distribution } \\
\text { of UDP-D-glucose from }\end{array}$ \\
& enzymatic conversion in excess $\mathrm{D}_{2} \mathrm{O}$ & enzymatic conversion in $\mathrm{H}_{2} \mathrm{O}$ \\
\hline 565 & 1 & 1 \\
566 & 0.33 & 0.189 \\
567 & 0.08 & 0.053 \\
\hline
\end{tabular}

retention time ( $\sim 40 \mathrm{pmol})$ (Fig. 6e), eluting between the substrate UDP-D-glucose (3.2 $\mathrm{min})$ and UDP-D-glucose5,6-ene (3.8 $\mathrm{min})$. MS analysis of the new reaction product yielded a molecular mass of $563.084 \mathrm{u}$ (ESI-MS analysis in the negative mode; Fig. 6e). This value obviously corresponds to the molecular mass of oxidized UDP-D-glucose. The accumulation of the new product was only detectable at high Ag13 concentration. The putative keto-compound remained tightly associated with the active site of Agl3 during substrate turnover, and could be extracted only by instantaneous chloroform-induced unfolding of Agl3 during the catalytic conversion of UDP-D-glucose to UDP-Dglucose-5,6-ene (Sporty et al. 2008). Because of the fast rate of conversion, the accumulation of the putative ketocompound is very low, thus preventing structural analysis by NMR. The existence of a 4-keto product in the reaction pathway of different SDR enzymes was shown in plants (Pugh et al. 1995; Mulichak et al. 1999) and for the model enzymes GalE (Thoden et al. 1996a, b) and RmlB
(Allard et al. 2002). Currently it is not possible to continuously monitor every step of the formation of UDP-sulfoquinovose and thus, the overall mechanism of this bi-substrate enzyme is not yet fully understood.

Conversion of UDP-D-glucose by Agl3, analyzed after deuterium labeling

The conversion of UDP-D-glucose to UDP-D-glucose-5,6ene at an excess of $\mathrm{D}_{2} \mathrm{O}$ (Fig. 7) indicated enolization and locking of deuterium at C-5 of UDP-D-glucose (Gross et al. 2000). For this approach, purified Agl3 was dialyzed against $20 \mathrm{mM}$ phosphate buffer of $\mathrm{pD}$ 6.4. Substrate conversion in $\mathrm{D}_{2} \mathrm{O}$-phosphate buffer was repeated several times to obtain sufficient material for the subsequent ESI-MS analysis. As a result, $15 \%$ of UDP-D-glucose were labeled with one deuterium atom, with the ratio of isotope distribution in UDP-D-glucose-5,6-ene after enzymatic conversion in $\mathrm{D}_{2} \mathrm{O}$-phosphate remaining unchanged compared to the 
Fig. 8 Proposed mechanism for the conversion of UDPD-glucose to UDP-D-glucose5,6-ene (a) NAD-dependent oxidation of UDP-D-glucose at C-4 (structure 3), enolisation of UDP-4-keto-glucose (structure 4) and locking of deuterium at $\mathrm{C}-5$ by the enolisation process at the active site of $\mathrm{Agl} 3$ (structures 5-7). b Proposed NAD-dependent dehydration of the enol-conformation of UDP-4-keto-glucose eventually resulting in UDP-D-glucose-5,6ene. $160 \times 135 \mathrm{~mm}(300 \times 300$ DPI)
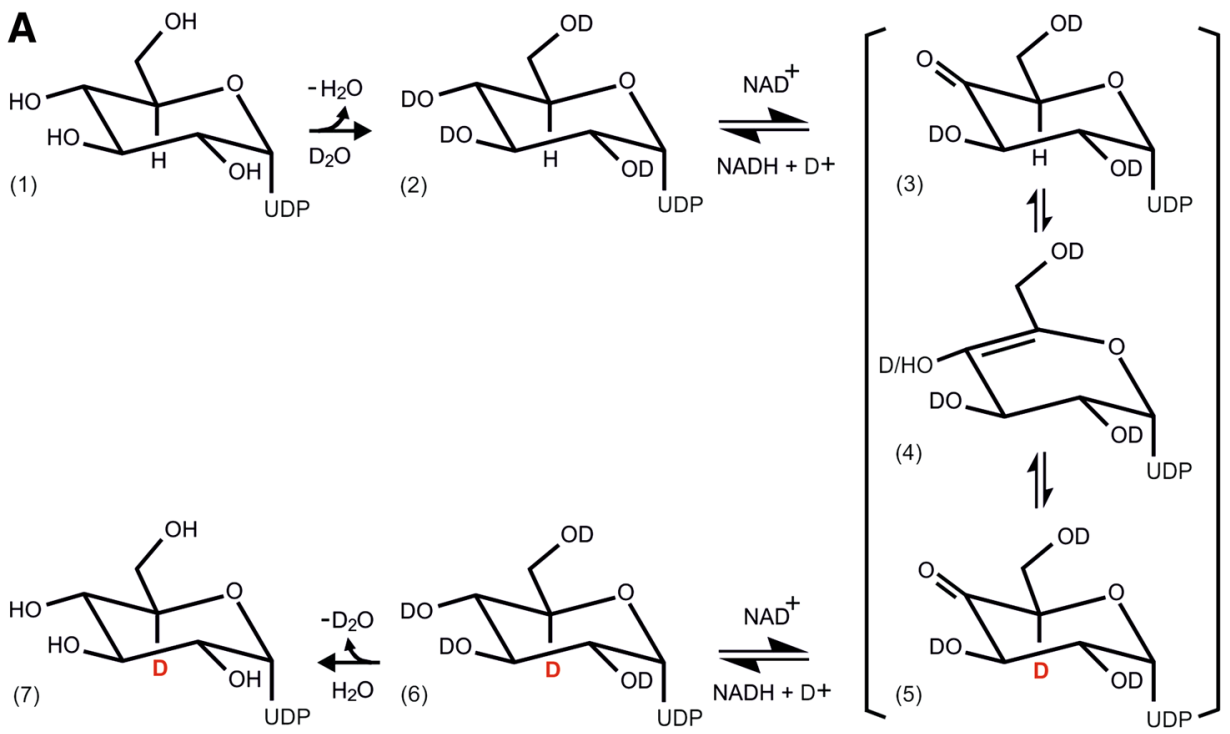

(5)

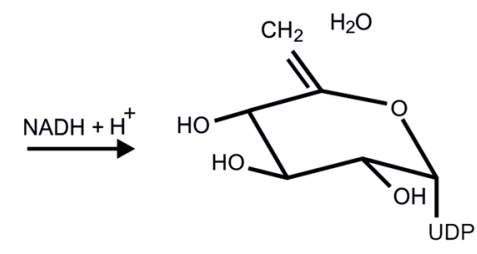

substrate (Fig. 7; Table 4). This indicated that the altered isotope distribution was specific for UDP-D-glucose and not the result of a deuterium contamination. Additionally, the stability of UDP-D-glucose in $\mathrm{D}_{2} \mathrm{O}$-phosphate buffer was tested, confirming that in the absence of Agl3, UDPD-glucose remained stable and unlabeled (not shown). Interestingly, the catalytic conversion of UDP-D-glucose to UDP-D-glucose-5,6-ene in $\mathrm{D}_{2} \mathrm{O}$-phosphate buffer increased to nearly $50 \%$ compared to the conversion in $\mathrm{H}_{2} \mathrm{O}(\sim 10 \%)$ (Fig. 7a, b).

The assumed keto-enol tautomeric equilibrium after oxidation of the $\mathrm{OH}$ group at C-4 of UDP-D-glucose was attempted to be shown by deuterium labeling of the substrate at the active site of Agl3 (Gross et al. 2000) (Fig. 8). This inter-conversion requires the release of the hydrogen at C-5 of the putative UDP-4-keto-glucose (Fig. 8a, structure 3 ) and subsequent formation of a double bond between C-4 and C-5 (Fig. 8a, structure 4). The inter-conversion of the putative UDP-4-keto-glucose and its enol form directly at the active site of $\mathrm{Agl} 3$ was traced by locking a deuterium atom at the $\mathrm{C}-5$ position of the substrate by the enolization process (Fig. 8a, structures 4, 5), and labeling of UDP-D-glucose with deuterium when the conversion of UDP-D-glucose by Agl3 was performed at an excess of $\mathrm{D}_{2} \mathrm{O}$ (Fig. 8a, structure 6). Labeling of C-5 with deuterium (e.g. C-5 of dTDP-D-glucose of RmlB) has been proposed for nucleotide-activated glucose intermediates involving keto-formation at C-4 followed by enolization between C-4 and C-5 (Gross et al. 2000). The observed labeling of UDPD-glucose with deuterium (Fig. 8a, structures 6,7) also suggests that dehydration of UDP-D-glucose occurs only from the enol form of UDP-4-keto-glucose (Fig. 8a, structure 4). This does explain why UDP-D-glucose-5,6-ene at C-5 could not be labeled with deuterium (Fig. 8b; Table 4). Deuterium is cleaved off from UDP-4-keto-glucose at the active site of Agl3 prior to its dehydration (Fig. 8a, interconversion of structure 5 to 4 ). Thus, dehydration of UDP4-keto-glucose is proposed to occur through the enol form (Fig. 8b), followed by the transfer of hydride to C-4. The assumed hydride transfer diverts here from the common dehydratase pathway, where hydride transfer to C-6 has been proposed (Field and Naismith 2003). This step might be accomplished without the suggested sugar rotation and still keep C-4 in an optimal position for hydride transfer. It would allow sulfite addition to an enone for subsequent UDP-sulfoquinovose biosynthesis (Fig. 9) but we cannot structurally prove our proposal because of the current lack of a crystal structure of Agl3.

An interesting observation concerns the nearly $50 \%$ increase of the catalytic conversion of UDP-D-glucose to UDP-D-glucose-5,6-ene in $\mathrm{D}_{2} \mathrm{O}$-phosphate buffer when compared to the conversion in $\mathrm{H}_{2} \mathrm{O}$-phosphate buffer (Fig. 8). There is sufficient volume for a significant amount of water molecules to be present at the active site of $\mathrm{Agl} 3$ 
Fig. 9 Comparison of reaction mechanisms of classical 4,6-dehydratases (Field and Naismith 2003) and the proposed reaction mechanisms of $\mathrm{Agl} 3$ in the absence and presence of sulfite resulting either in the formation of UDPD-glucose-5,6-ene or UDPsulfoquinovose. $161 \times 197 \mathrm{~mm}$ $(300 \times 300 \mathrm{DPI})$

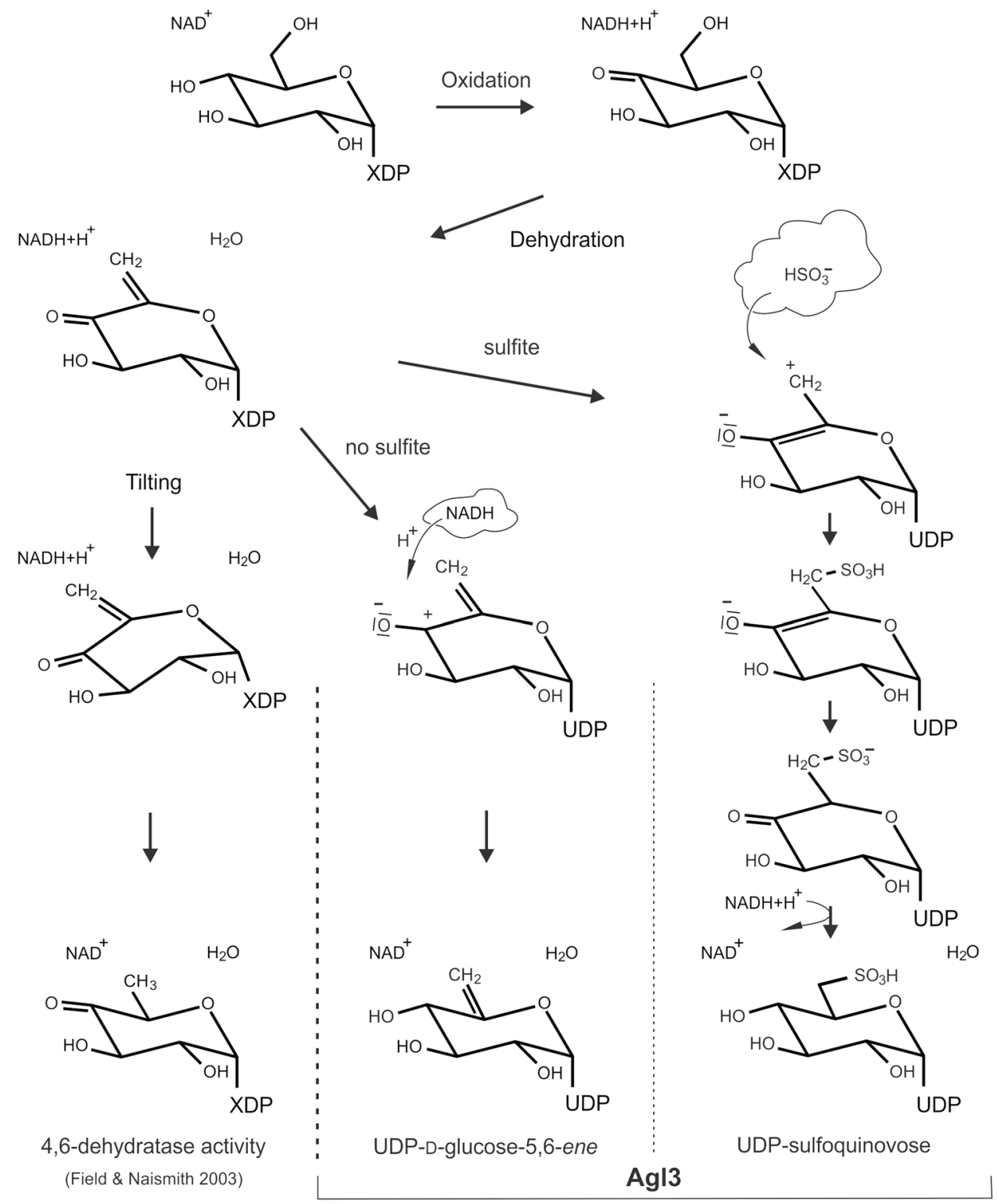

mechanism of this subfamily. This proof is particularly important because of the possible distant evolutionary relationship between Ag13 and SQD1 from A. thaliana (Fig. 1a).

If the reaction mechanisms of Agl3 and SQD1 are indeed different, other crucial amino acids than those identified for SQD1 should be involved in the catalytic reaction. This implicates presumably a dehydratase reaction in Agl3 (Allard et al. 2002; Field and Naismith 2003). The respective catalytic tyrosine residue in Agl3 could be Tyr148 (Figs. 1, 2a, b). It might function as active site base and accomplish - in concert with Glu147-the dehydration step (Allard et al. 2002). With the RmlB enzyme from Salmonella enterica sv. Typhimurium it was shown that the C-4 of the substrate was at the optimal position for initial hydride abstraction (Allard et al. 2002). The results nism of Agl3 of $S$ acidocaldarius follows the general 
obtained with Agl3 are in agreement with this reaction step (Fig. 9), in which $\mathrm{NAD}^{+}$initially oxidizes glucosyl C-4 of dTDP-glucose to dTDP-4-keto-glucose, leaving the NADcofactor reduced. Next, water is eliminated between C-5 and C-6 of dTDP-4-keto-glucose to form dTDP-4-ketoglucose-5,6-ene. Hydride transfer from NADH to C-6 of dTDP-4-keto-glucose-5,6-ene regenerates $\mathrm{NAD}^{+}$and produces the product dTDP-4-keto-6-deoxyglucose (Gross et al. 2000). It was proposed that water remains bound to the protein and sugar rotation around the glycosidic linkage creates no steric clash but should move C-6 to an appropriate location for accepting the hydride (Field and Naismith 2003).

The reaction pathway of the UDP-sulfoquinovose synthase Agl3 of S. acidocaldarius obviously follows the general pathway described for dehydratases (Field and Naismith 2003), but seems to be modified at the final step (Fig. 9). Based on our data we assume the final hydride transfer to occur to C-4 rather than C-6 of the enone form of UDP-4-keto-glucose, leaving this carbon accessible for the subsequent addition of sulfite (Fig. 9). Conclusive information about the actual reaction mechanism of Agl3 is expected from $3 \mathrm{D}$ crystallization experiments which are currently in progress.

\section{Procedures}

Agl3 expression and point mutation analysis

Recombinant production and nickel affinity purification of hexa-histidine-tagged Agl3 from S. acidocaldarius were performed as described previously (Meyer et al. 2011). Point mutations were performed by overlap-extension PCR. Ten amino acid positions in Agl3 were selected on the basis of critical amino acids either from epimerases or dehydratases (Mulichak et al. 1999; Field and Naismith 2003). Targeted amino acids in Agl3 along with the primers carrying the alanine codon for replacement of the selected codon are shown in Table 1, 2. First, agl3 was cloned into pET30a (Novagen), using the primer pair 3'-CCCCCC CATATGAGGATTCTAGTACTAGGAATT-5'/3'-CCCCCC CTCGAGACCACCCGCACCACCTCTTACTCTTTTAAC GTATTGTGGTTT-5'. The first phase of overlap-extension PCR was performed with $7 \mathrm{ng}$ of pET30_Agl3 as a template, one unit of Phusion polymerase (Fermentas), $10 \mathrm{mM}$ of dNTP mix and amplification conditions as follows: one cycle at $94{ }^{\circ} \mathrm{C}$ for $4 \mathrm{~min}$, followed by 30 cycles at $94{ }^{\circ} \mathrm{C}$ for $30 \mathrm{~s}$, each, for denaturation, and annealing at $55{ }^{\circ} \mathrm{C}$ for $30 \mathrm{~s}$, extension/elongation at $72{ }^{\circ} \mathrm{C}$ for $90 \mathrm{~s}$, and one final elongation cycle at $72{ }^{\circ} \mathrm{C}$ for $5 \mathrm{~min}$. The amplification products were purified using the gel extraction kit from Fermentas and used as templates (approximately $5 \mathrm{ng}$, each) for the second phase of the overlap-extension PCR using identical amplification conditions. The amplification products were purified (see above) and cloned into pET30a via the NdeI and XhoI restriction sites (Meyer et al. 2011). The point mutations were confirmed by single-run sequencing (LGC genomics); the Agl3 proteins carrying the different alanine mutations were expressed in E. coli BL21 DE3 cells (Stratagene) after induction with $1 \mathrm{mM}$ isopropyl- $\beta$ D-thiogalactopyranoside (IPTG) and purified as described for Agl3 (Meyer et al. 2011).

Agl3 in vitro assay and saturation kinetics measurements

The conversion of UDP-D-glucose to UDP-D-glucose5,6-ene catalyzed by $\mathrm{Agl} 3$ was performed in $20 \mathrm{mM}$ 1,3-bis(tris-(hydroxymethyl)methylamino)propane (BisTris propane) buffer, $\mathrm{pH}$ 6.5, containing UDP-D-glucose at a final concentration of $0.1,0.2,0.5,1.0,2.0,4.0$, and $10 \mathrm{mM}$, respectively, and $20 \mu \mathrm{g}$ of purified $\mathrm{Agl} 3$ in a total volume of $40 \mu \mathrm{l}$ of reaction mixture. The reaction was carried out for $30 \mathrm{~min}$ at $70{ }^{\circ} \mathrm{C}$. Subsequently, the reaction mixture was rapidly cooled on ice and both the substrate and reaction product were extracted by the addition of $40 \mu \mathrm{l}$ of chloroform followed by vortexing the mixture for $1 \mathrm{~min}$. Phase separation was performed using a table centrifuge (Eppendorf 5804R) and the water phase was analyzed by RP-HPLC (Thermo Scientific/Dionex; Ultimate 3000 Standard LC System) on a Nucleosil 120-3 C18 column (Macherey-Nagel) with a flow rate of $0.6 \mathrm{ml} \mathrm{min}{ }^{-1}$ and $0.4 \mathrm{M}$ phosphate (pH 6.1) (Meyer et al. 2011). The formation of UDP-D-glucose-5,6-ene was investigated by PGCESI-MS(MS) analysis after pre-purification of the intermediate on a porous graphitized carbon (PGC) cartridge (Thermo Scientific) (Pabst et al. 2010). The conversion of UDP-D-glucose and sulfite to UDP-sulfoquinovose by Agl3 was measured under the same conditions by varying UDPD-glucose concentrations of $0.1,0.3,1,3,10$ and $30 \mathrm{mM}$ against sodium sulfite concentrations of $1.0,3.0,10,30$, and $100 \mathrm{mM}$.

PGC purification and desalting of UDP-activated sugars

Prior to analysis of the in vitro reaction products of $\mathrm{Agl} 3$ by NMR and ESI-MS(MS), the UDP-bound intermediates from the reaction mixture were made protein-free and desalted using PGC spin-prep columns (Thermo Scientific). The columns were pre-activated with $500 \mu \mathrm{l}$ of $100 \%$ acetonitrile and washed with $500 \mu \mathrm{l}$ of MilliQ water prior to sample application. Bound UDP-sugars were eluted with $100 \%$ acetonitrile and lyophilized on a SpeedVac vacuum concentrator (Pabst et al. 2010). For ESI-MS(MS) analysis, the UDP-sugars were re-dissolved in $0.3 \mathrm{M}$ ammonium formate, $\mathrm{pH} 9$, containing $50 \%$ acetonitrile. The samples were 
measured via direct infusion on a Bruker maXis $4 \mathrm{G}$ mass spectrometer in the negative ion MS scan mode. Specific values were set to: spectra rate $1.0 \mathrm{~Hz}$, low mass $300 \mathrm{~m} / \mathrm{z}$, ion transfer time $85 \mu \mathrm{s}$, pre pulse storage $10.0 \mu \mathrm{s}$.

NMR analysis of the Agl3 product

Spectra were recorded at $297 \mathrm{~K}$ in $99.9 \% \mathrm{D}_{2} \mathrm{O}(0.6 \mathrm{ml})$ on an Avance III 600 spectrometer (Bruker; ${ }^{1} \mathrm{H}$ at $600.13 \mathrm{MHz},{ }^{13} \mathrm{C}$ at $150.9 \mathrm{MHz},{ }^{31} \mathrm{P}$ at $242.9 \mathrm{MHz}$ ), using standard Bruker NMR software. ${ }^{1} \mathrm{H}$ NMR spectra were referenced to 2,2-dimethyl-2-silapentane-5-sulfonic acid $(\delta 0.0),{ }^{13} \mathrm{C}$ NMR spectra were referenced to external dioxane $(\delta 67.40)$, and ${ }^{31} \mathrm{P}$ spectra were referenced to external ortho-phosphoric acid $(\delta 0.0)$ for solutions in $\mathrm{D}_{2} \mathrm{O}$. Gradient-selected ${ }^{1} \mathrm{H},{ }^{1} \mathrm{H}$ total correlated spectroscopy (TOCSY, mixing time $120 \mathrm{~ms}$ ) and COSY experiments were recorded using the programs mlevph and cosygpqf, respectively, with $2048 \times 256$ data points and 16 and 8 scans, respectively per $t 1$-increment. The multiplicity edited heteronuclear single quantum coherence spectra (HSQC) (Schleucher et al. 1994) were measured using the program hsqcedetgp with $1024 \times 128$ data points and 128 scans per t1-increment. Heteronuclear multiple bond correlation spectra (HMBC) (Bax and Summers 1986) were acquired using the pulse program hmbcgpndqf with $4096 \times 64$ data points and 1600 scans per t1-increment and spectral widths of $7.7 \mathrm{ppm}$ for ${ }^{1} \mathrm{H}$ and $222 \mathrm{ppm}$ for ${ }^{13} \mathrm{C}$ to check for any carbonyl correlated signals.

\section{Labeling with deuterium oxide}

The conversion of UDP-D-glucose to UDP-D-glucose-5,6ene was carried out in $20 \mathrm{mM}$ phosphate buffer, prepared in $\mathrm{D}_{2} \mathrm{O}$, at a $\mathrm{pD}$ value of 6.4 (Gabriel and Lindquist 1968). Briefly, an acidic and a basic phosphate stock solution in $\mathrm{D}_{2} \mathrm{O}$ were prepared by dissolving $0.037 \mathrm{~g}$ of $\mathrm{Na}_{2} \mathrm{HPO}_{4}$ and $0.035 \mathrm{~g}$ of $\mathrm{NaH}_{2} \mathrm{PO}_{4}$ in $10 \mathrm{ml}$ of $\mathrm{D}_{2} \mathrm{O}$, each, at a final concentration of $20 \mathrm{mM}$, and the $\mathrm{pD}$ values of $\mathrm{D}_{2} \mathrm{O}$-prepared phosphate solutions were determined using a $\mathrm{pH}$ meter (Mettler MP-220); approximate values were 5.3 and 8.8 for the acidic and the basic phosphate stock solution, respectively. The acidic and basic phosphate stock solutions were mixed at a ratio of $3: 1(\mathrm{v} / \mathrm{v})$ to obtain a $20 \mathrm{mM}$ phosphate buffer in $\mathrm{D}_{2} \mathrm{O}$ in the range of $\mathrm{pD}$ 6.4. For maximum removal of protons from the phosphate solutions, the solvents were dried using the SpeedVac concentrator and either phosphate salt was re-dissolved in $1 \mathrm{ml}$ of $\mathrm{D}_{2} \mathrm{O}$. $\mathrm{Agl} 3$ was prepared in $\mathrm{D}_{2} \mathrm{O}$ as follows: $2 \mathrm{ml}$ of $\mathrm{Agl} 3$ solution (1 $\mathrm{mg}$ protein), purified by nickel affinity chromatography and dialyzed against $10 \mathrm{mM}$ phosphate buffer, $\mathrm{pH}$ 6.5, were concentrated to $100 \mu \mathrm{l}$ using the SpeedVac concentrator and supplemented with $\mathrm{D}_{2} \mathrm{O}$ to a final volume of $1 \mathrm{ml}$, corresponding to a protein concentration of $1 \mathrm{mg} \mathrm{ml}^{-1}$.

Cofactor extraction and spectrophotometric analysis

Purified Agl3 (5.6 mg of protein in $10 \mathrm{ml}$ of $5 \mathrm{mM}$ phosphate buffer, $\mathrm{pH} 6.5$ ) was added to $5 \mathrm{ml}$ of chloroform and mixed for $1 \mathrm{~h}$ at room temperature $\left(22^{\circ} \mathrm{C}\right)$. Phase separation was done using a table centrifuge and the water phase was transferred to a new tube. Washing of the chloroform phase was repeated with $2 \mathrm{ml}$ of MilliQ water. The combined water phases were concentrated to $1 \mathrm{ml}$ (SpeedVac) and analyzed for the presence of the NAD-cofactor by RP-HPLC (as described above) (Meyer et al. 2011). The absorption spectrum of fractions containing $\mathrm{NAD}^{+}$ was measured on a diode array photometer (Agilent) in $100-\mu l$ quartz cuvettes. The in vitro reduction of extracted $\mathrm{NAD}^{+}$was performed qualitatively by supplementing trace amounts of glucose dehydrogenase (Amano) and glucose (1 mM final concentration) to the NAD-cofactor and incubating the mixture at room temperature for 2 and $5 \mathrm{~min}$, respectively.

\section{Conventional stopped-flow spectroscopy}

Transient-state measurements were made using the SX.18 MV stopped-flow spectrophotometer (Applied Photophysics, Leatherhead, Surrey, UK), equipped with a $1 \mathrm{~cm}$ observation cell. In a typical experiment, UDP-sulfoquinovose synthase was mixed with UDP-D-glucose at $65{ }^{\circ} \mathrm{C}$ and first data points recorded starting with $1.5 \mathrm{~ms}$. Final concentrations were $2 \mu \mathrm{M}$ of $\mathrm{Ag} 13$ and $0.5,5,25$ and $50 \mu \mathrm{M}$ of UDP-D-glucose, respectively. The reduction of $\mathrm{NAD}^{+}$ was followed at $320 \mathrm{~nm}$. Calculation of pseudo-first-order rate constants $\left(k_{\mathrm{obs}}\right)$ from experimental time traces was performed with a SpectraKinetic work station (Version 4.38) interfaced to the instruments. The second-order rate constant was calculated from the slope of the linear plot of the pseudo-first-order rate constants versus substrate concentration. To follow spectral transitions, a Model PD.1 photodiode array accessory (Applied Photophysics) connected to the stopped-flow machine together with XScan diode array scanning software (Version 1.07) was utilized.

\section{Circular dichroism (CD) measurements}

Electronic circular dichroism spectroscopy was performed using Chirascan (Applied Photophysics, Leatherhead, UK). First, the instrument was flushed with nitrogen at a flow rate of $51 \mathrm{~min}^{-1}$. Then, ECD spectra were recorded at room temperature in the far-UV region (i.e., $180-260 \mathrm{~nm}$ ). The path length was $1 \mathrm{~mm}$, spectral band width $3 \mathrm{~nm}$ and scan time per point $10 \mathrm{~s}$. 
Acknowledgments This work was supported by the Austrian Science Fund FWF, projects P22791-B11 and P24305-B20 to PM, $\mathrm{P} 21954-\mathrm{B} 20$ and $\mathrm{P} 24307-\mathrm{B} 22$ to CS, and the PhD program "BioToPBiomolecular Technology of Proteins" (Austrian Science Fund FWF, project W1224, speaker COb).

Open Access This article is distributed under the terms of the Creative Commons Attribution License which permits any use, distribution, and reproduction in any medium, provided the original author(s) and the source are credited.

\section{References}

Allard STM, Beis K, Giraud M-F, Hegeman AD, Gross JW, Wilmouth RC, Whitfield C, Graninger M, Messner P, Allen AG, Maskell DJ, Naismith JH (2002) Toward a structural understanding of the dehydratase mechanism. Structure 10:81-92

Bax A, Summers MF (1986) ${ }^{1} \mathrm{H}$ and ${ }^{13} \mathrm{C}$ assignments from sensitivity-enhanced detection of heteronuclear multiple-bond connectivity by $2 \mathrm{D}$ multiple quantum NMR. J Am Chem Soc 108:2093-2094

Benning C (1998) Biosynthesis and function of the sulfolipid sulfoquinovosyl diacylglycerol. Annu Rev Plant Physiol Plant Mol Biol 49:53-75

Benning C, Beatty JT, Prince RC, Somerville CR (1993) The sulfolipid sulfoquinovosyl diacylglycerol is not required for photosynthetic electron transport in Rhodobacter sphaeroides but enhances growth under phosphate limitation. Proc Natl Acad Sci USA 90:1561-1565

Bolhuis H, Palm P, Wende A, Falb M, Rampp M, Rodriguez-Valera F, Pfeiffer F, Oesterhelt D (2006) The genome of the square archaeon Haloquadratum walsbyi: life at the limits of water activity. BMC Genomics 7:169-180

Denger K, Weiss M, Felux A-K, Schneider A, Mayer C, Spiteller D, Huhn T, Cook AM, Schleheck D (2014) Sulphoglycolysis in Escherichia coli $\mathrm{K}-12$ closes a gap in the biogeochemical sulphur cycle. Nature 507:114-117

Eichler J (2013) Extreme sweetness: protein glycosylation in archaea. Nat Rev Microbiol 11:151-156

Essigmann B, Hespenheide BM, Kuhn LA, Benning C (1999) Prediction of the active-site structure and $\mathrm{NAD}^{+}$binding in SQD1, a protein essential for sulfolipid biosynthesis in Arabidopsis. Arch Biochem Biophys 369:30-41

Field RA, Naismith JH (2003) Structural and mechanistic basis of bacterial sugar nucleotide-modifying enzymes. Biochemistry 42:7637-7647

Gabriel O, Lindquist LC (1968) Biological mechanisms involved in the formation of deoxy sugars. IV. Enzymatic conversion of thymidine diphosphoglucose- $4 T$ to thymidine diphospho-4-keto6-deoxyglucose-6T. J Biol Chem 243:1479-1484

Gross JW, Hegeman AD, Vestling MM, Frey PA (2000) Characterization of enzymatic processes by rapid mix-quench mass spectrometry: the case of dTDP-glucose 4,6-dehydratase. Biochemistry 39:13633-13640

Gross JW, Hegeman AD, Gerratana B, Frey PA (2001) Dehydration is catalized by glutamate-136 and aspartic acid-135 active site residues in Escherichia coli dTDP-glucose 4,6-dehydratase. Biochemistry 40:12497-12504

Hegeman AD, Gross JW, Frey PA (2001) Probing catalysis by Escherichia coli dTDP-glucose-4,6-dehydratase: identification and preliminary characterization of functional amino acid residues at the active site. Biochemistry 40:6598-6610

Kavanagh KL, Jörnvall H, Persson B, Oppermann U (2008) Mediumand short-chain dehydrogenase/reductase gene and protein families: the SDR superfamily: functional and structural diversity within a family of metabolic and regulatory enzymes. Cell Mol Life Sci 65:3895-3906

Kwei CK, Lewis DM, King KD, Donohue W, Neilan BA (2011) Molecular classification of commercial Spirulina strains and identification of their sulfolipid biosynthesis genes. J Microbiol Biotechnol 21:359-365

Li T, Simonds L, Kovrigin EL, Noel KD (2014) In vitro biosynthesis and chemical identification of UDP- $N$-acetyl-D-quinovosamine (UDP-D-QuiNAc). J Biol Chem 289:18110-18120

Liu Y, Thoden JB, Kim J, Berger E, Gulick AM, Ruzicka FJ, Holden HM, Frey PA (1997) Mechanistic roles of tyrosine 149 and serine 124 in UDP-galactose 4-epimerase from Escherichia coli. Biochemistry 36:10675-10684

Meyer BH, Zolghadr B, Peyfoon E, Pabst M, Panico M, Morris HR, Haslam SM, Messner P, Schäffer C, Dell A, Albers S-V (2011) Sulfoquinovose synthase - an important enzyme in the $\mathrm{N}$-glycosylation pathway of Sulfolobus acidocaldarius. Mol Microbiol 82:1150-1163

Meyer BH, Peyfoon E, Dietrich C, Hitchen P, Panico M, Morris HR, Dell A, Albers S-V (2013) Agl16, a thermophilic glycosyltransferase mediating the last step of $\mathrm{N}$-glycan biosynthesis in the thermoacidophilic crenarchaeon Sulfolobus acidocaldarius. J Bacteriol 195:2177-2186

Mulichak AM, Theisen MJ, Essigmann B, Benning C, Garavito RM (1999) Crystal structure of SQD1, an enzyme involved in the biosynthesis of the plant sulfolipid headgroup donor UDP-sulfoquinovose. Proc Natl Acad Sci USA 96:13097-13102

Mulichak AM, Bonin CP, Reiter W-D, Garavito RM (2002) Structure of the MUR1 GDP-mannose 4,6-dehydratase from Arabidopsis thaliana: implications for ligand binding and specificity. Biochemistry 41:15578-15589

Pabst M, Grass J, Fischl R, Léonard R, Jin C, Hinterkörner G, Bort N, Altmann F (2010) Nucleotide and nucleotide sugar analysis by liquid chromatography-electrospray ionization-mass spectrometry on surface-conditioned porous graphitic carbon. Anal Chem 82:9782-9788

Parente J, Casabuono A, Ferrari MC, Paggi RA, De Castro RE, Couto AS, Giménez MI (2014) A rhomboid protease gene deletion affects a novel oligosaccharide $N$-linked to the S-layer glycoprotein of Haloferax volcanii. J Biol Chem 289:11304-11317

Peyfoon E, Meyer B, Hitchen PG, Panico M, Morris HR, Haslam SM, Albers S-V, Dell A (2010) The S-layer glycoprotein of the crenarchaeote Sulfolobus acidocaldarius is glycosylated at multiple sites with chitobiose-linked $N$-glycans. Archaea 2010, article ID 754101

Pugh CE, Roy AB, Hawkes T, Harwood JL (1995) A new pathway for the synthesis of the plant sulpholipid, sulphoquinovosyldiacylglycerol. Biochem J 309:513-519

Riekhof WR, Ruckle ME, Lydic TA, Sears BB, Benning C (2003) The sulfolipids 2'-O-acyl-sulfoquinovosyldiacylglycerol and sulfoquinovosyldiacylglycerol are absent from a Chlamydomonas reinhardtii mutant deleted in SQD1. Plant Physiol 133:864-874

Sanda S, Leustek T, Theisen MJ, Garavito RM, Benning C (2001) Recombinant Arabidopsis SQD1 converts UDP-glucose and sulfite to the sulfolipid head group precursor UDP-sulfoquinovose in vitro. J Biol Chem 276:3941-3946

Sato N, Sugimoto K, Meguro A, Tsuzuki M (2003) Identification of a gene for UDP-sulfoquinovose synthase of a green alga, Chlamydomonas reinhardtii, and its phylogeny. DNA Res 10:229-237

Schleucher J, Schwendinger M, Sattler M, Schmidt P, Schedletzky O, Glaser SJ, Sørensen OW, Griesinger C (1994) A general enhancement scheme in heteronuclear multidimensional NMR employing pulsed field gradients. J Biomol NMR 4:301-306

Shimojima M (2011) Biosynthesis and functions of the plant sulfolipid. Progr Lipid Res 50:234-239 
Shimojima M, Hoffmann-Benning S, Garavito RM, Benning C (2005) Ferredoxin-dependent glutamate synthase moonlights in plant sulfolipid biosynthesis by forming a complex with SQD1. Arch Biochem Biophys 436:206-214

Somoza JR, Menon S, Schmidt H, Joseph-McCarthy D, Dessen A, Stahl ML, Somers WS, Sullivan FX (2000) Structural and kinetic analysis of Escherichia coli GDP-mannose 4,6 dehydratase provides insights into the enzyme's catalytic mechanism and regulation by GDP-fucose. Structure 8:123-135

Sporty JL, Kabir MM, Turteltaub KW, Ognibene T, Lin SJ, Bench G (2008) Single sample extraction protocol for the quantification of NAD and NADH redox states in Saccharomyces cerevisiae. J Sep Sci 31:3202-3211
Thoden JB, Frey PA, Holden HM (1996a) High-resolution X-ray structure of UDP-galactose 4-epimerase complexed with UDPphenol. Protein Sci 5:2149-2161

Thoden JB, Frey PA, Holden HM (1996b) Molecular structure of the NADH/UDP-glucose abortive complex of UDP-galactose 4-epimerase from Escherichia coli: implications for the catalytic mechanism. Biochemistry 35:5137-5144

Zähringer U, Moll H, Hettmann T, Knirel YA, Schäfer G (2000) Cytochrome $b_{558 / 566}$ from the archaeon Sulfolobus acidocaldarius has a unique Asn-linked highly branched hexasaccharide chain containing 6-sulfoquinovose. Eur J Biochem 267:4144-4149 